\title{
A Kinetic Model for Gas absorption-Desorption at Solid Surfaces Under Non-Equilibrium Conditions
}

\author{
Ricardo D. Brancher, Stefan Stefanov, Irina Graur, Aldo Frezzotti \\ ${ }^{1}$ Aix Marseille Université, CNRS, IUSTI UMR 7343, 5 rue E. Fermi, 13453, Marseille, France \\ 2 Institute of Mechanics, BASci., Acad. G. Bonchev Str., Block. 4, 1113, Sofia, Bulgaria \\ ${ }^{3}$ Dipartimento di Scienze $\&$ Tecnologie Aerospaziali del Politecnico di Milano, Via La Masa 34, 20156, Milano, Italy
}

\begin{abstract}
One-dimensional flows of a polyatomic gas in the presence of an adsorbing-desorbing surface kept at constant and uniform temperature are simulated by solving numerically the Boltzmann kinetic equation by the Direct Simulation Monte Carlo (DSMC) method. It is considered the flow of gas between two planar and infinite surfaces, where only one surface is able to adsorb and desorb molecules, while the other one is impermeable. Borgnakke-Larsen model is used to describe inelastic collisions dynamics for a gas of rigid rotators. The absorption and desorption phenomena are simulated by a kinetic model which includes the classical Langmuir description as a limit case and leads to an additional equation for the time evolution of the surface coverage to be solved along with the gas dynamics. Furthermore, the analytical solution is developed for free molecular flow regime. The comparison between the analytical solution and the results obtained by the numerical solution for this regime shows a very good agreement.
\end{abstract}

Email addresses: ricardo.d.brancher@gmail.com (Ricardo D. Brancher), stefanov@imbm.bas.bg (Stefan Stefanov), irina.martin@univ-amu.fr (Irina Graur), aldo.frezzotti@polimi.it (Aldo Frezzotti) 


\section{INTRODUCTION}

The gas flows induced or affected by mass transfer of gaseous species onto (or from) solid surfaces (absorption and desorption) are present in many natural, physical, biological, and chemical systems and are widely used in different industrial applications $[1,2,3]$, such as water purification, air conditioning (absorption chillers), vapor deposition (CD/DVD metallization) and vacuum pumping (getters and cryogenic pumps). The absorption and desorption phenomena are also used for the gas separation in chromatographic columns, where different species of a gaseous mixture can be separated due to their different residence times. Activated carbons are the most often used adsorbent type, although there are other adsorbent types with pores size of molecular dimensions, like zeolites, which give them a certain selectivity for molecules of the specific size and shape.

To simulate the absorption-desorption phenomena the boundary condition for the gas in contact with the absorbing-desorbing surface have to be formulated. Currently, around fifteen different absorptiondesorption models can be found in the literature. The most used ones among them are based on Henry's [4] and Langmuir's absorption isotherms [5] and the BET absorption theory [6]. In the frame of the two simplest models, Henry's and Langmuir's, the formation of a monolayer on a solid surface is assumed, while the BET model allows the formation of a multilayer. However, all these models are based on the equilibrium assumption, i.e. the absorption and desorption fluxes are in equilibrium.

In the present work we propose a new model of the absorption-desorption phenomena which is based on the description of the gas flow at molecular level. The gas flow between the surfaces is simulated using the classical DSMC method [7], where the intermolecular collisions are calculated using the BorgnakkeLarsen model [? ]. The evaluation of the absorption and desorption fluxes is based on the kinetic flow description including the absorption probability. The proposed model was applied for the first time to simulate the flow in the vicinity of a solid surface [8]. In the present paper this new model was improved and used for the simulation of the gas flow between two parallel plates, where one of them could adsorb and desorb the molecules and the other one only reflects them. The influence of the gas rarefaction, heat of absorption, residence time and absorption probability on the surface coverage is evaluated. The surface coverage obtained in the frame of the proposed model is compared to that calculated by the classical Langmuir absorption isotherm.

\section{2. absorption AND DESORPTION ON THE SURFACE}

Let us start with a brief general conception of the largely used approach for the absorption and desorption phenomena simulation, the Langmuir theory of monolayer absorption [5].

\subsection{Langmuir absorption Isotherm}

According to Langmuir theory of absorption [5], a certain number of binding sites per unit area, $N_{\max }$, exists on a solid perfectly clean (bare) surface. Of these binding sites, $N$ are occupied with adsorbate and $N_{\max }-N$ are vacant. Based on that, the absorption rate is defined according to:

$$
R_{a d s}=K_{a d s} p\left(N_{\max }-N\right),
$$

where $p$ is the gas pressure near the adsorbed surface and $K_{a d s}$ is the absorption coefficient calculated as it suggested in Ref. [4]:

$$
K_{a d s}=\frac{\sigma A}{\sqrt{2 \pi m k_{B} T}}
$$


where $A$ is the area of one binding site, $\sigma$ is the absorption probability of a clean surface, $m$ is the molecular mass, $k_{\mathrm{B}}$ is the Boltzmann constant and $T$ is the surface temperature. The value of $A$ can be estimated based on the molecular diameter of an adsorbed gas molecule, and its typical value is $10^{-19} \mathrm{~m}^{2}$ [4]. It is worth to underline that the absorption rate, Eq. (1), depends on the pair gas and surface, i.e. on the gas pressure and temperature and on the surface nature and temperature, but also on the number of available sites for absorption, $N_{\max }-N$, and, finally, on the absorption probability. The desorption rate is calculated from:

$$
R_{d e s}=K_{d e s} N
$$

The desorption coefficient is defined as the inverse of absorption (or residence) time $\tau$ :

$$
K_{\text {des }}=\frac{1}{\tau},
$$

where

$$
\tau=\tau_{0} \exp \left(\frac{Q}{\mathcal{R}_{g} T}\right)
$$

In the previous equation, $\mathcal{R}_{g}=8.314 \mathrm{~J} \mathrm{~mol}^{-1} \mathrm{~K}^{-1}$ is the universal gas constant, $Q$ is the heat of absorption and the inverse of $\tau_{0}$ can be comprised as the surface bond vibration frequency. In the following text, this parameter, $\tau_{0}$, will be called residence time constant. The values of $\tau_{0}$ and $Q$, obtained experimentally for different pairs of gas-surface, can be found in the literature [4]. As the absorption rate, the desorption rate depends on the pair gas-surface, through the parameters $Q$ and $\tau_{0}$, but also on the number of gas molecules adsorbed on a surface, $N$.

In equilibrium, the absorption rate, Eq. (1), is equal to the desorption rate, Eq. (3), as:

$$
K_{a d s} p\left(N_{\max }-N\right)=K_{d e s} N
$$

otherwise a flux from (or into surface) may exist. By equalizing the absorption and desorption fluxes, Eq. (6), and dividing it by $N_{\max }$, we obtain the equilibrium between the fluxes in the form:

$$
K_{a d s} p(1-\theta)=K_{d e s} \theta
$$

From previous relation, the Langmuir isotherm is obtained:

$$
\theta=\frac{K_{L} p}{1+K_{L} p}
$$

where $\theta$ is the fractional surface coverage, defined as:

$$
\theta=\frac{N}{N_{\max }},
$$

and $K_{L}$ is the Langmuir constant calculated as:

$$
K_{L}=\frac{K_{a d s}}{K_{d e s}}=\frac{\sigma A \tau_{0}}{\sqrt{2 \pi m k_{B} T}} \exp \left(\frac{Q}{\mathcal{R}_{g} T}\right)
$$

To summarize, the absorption-desorption phenomena depend basically on four parameters: heat of absorption $(Q)$, residence time constant $\left(\tau_{0}\right)$, number of total active sites on the bare surface $\left(N_{\max }\right)$ and absorption probability $(\sigma)$. All these parameters depend on the specific nature of the solid surface and 
gas species involved in the process. The temperature of both gas and solid surface can also influence the absorption-desorption phenomena.

In view of the following discussion, it is convenient to reformulate the expression of $R_{a d s}$, given by Eq. (1), replacing the pressure $p$ by the impinging mass flux of the molecules to the adsorbed surface, $J_{m}$. In equilibrium, the latter is given by the following expression:

$$
J_{m}=n \sqrt{\frac{k_{B} T}{2 \pi m}},
$$

being $n$ the gas number density. Using Eq. (11), $R_{a d s}$ is written as:

$$
R_{a d s}=\sigma A J_{m}\left(N_{\max }-N\right) .
$$

The new form of $R_{a d s}$ keeps its validity out of equilibrium conditions, where the impinging mass flux $J_{m}$ cannot be related to pressure by Eq. (11).

\subsection{Non-Equilibrium Case}

Langmuir theory has been developed for the case of the equilibrium between absorption and desorption rates, Eq. (6), [4]. However, sometimes in practice, a net adsorbing (or desorbing) flux might exist, therefore the equality of absorption and desorption rates, Eq. (7), is not valid anymore and the surface coverage could vary as function of time. We assume here that the variation of the occupied binding sites, or surface number density, $N$, in time is proportional to the difference of the fluxes of adsorbed and desorbed molecules [9]:

$$
\frac{d N(t)}{d t}=K_{a d s} p\left(N_{\max }-N(t)\right)-K_{d e s} N(t)
$$

Previous equation can be divided by $N_{\max }$, so we obtain the equation for the variation of the surface coverage in time:

$$
\frac{d \theta(t)}{d t}=K_{a d s} p(1-\theta(t))-K_{d e s} \theta(t) .
$$

This equation can be solved analytically, under assumption that both absorption and desorption coefficients, calculated under equilibrium conditions, could also be used in this non-equilibrium case. In addition, we assume that the gas pressure is quasi constant during the considered time interval. This assumption is

justified in the case when the number of adsorbed (or desorbed) molecules is small compared to the total number of particles in a volume. However, it is not the case for the vacuum technology applications (low pressure) and also for the fast processes when a total pressure in a system changes rapidly. By integrating Eq. (14), assuming the pressure constancy, we obtain the analytical solution in following form:

$$
\theta(t)=\frac{p K_{L}}{1+p K_{L}}\left(1-\exp \left(-t / \tau_{\mathrm{e}}\right)\right)
$$

where

$$
\tau_{\mathrm{e}}=\frac{1}{K_{\text {ads }} p+K_{\text {des }}} .
$$

To obtain the previous relation we also assume that, initially, there are not adsorbed molecules on the surface (bare surface): $\theta(t=0)=0$. The time $\tau_{\mathrm{e}}$ can be presented in the following form:

$$
\tau_{\mathrm{e}}=\frac{\tau}{1+\tau / \tau_{\mathrm{a}}}, \quad \tau_{\mathrm{a}}=\frac{1}{K_{a d s} p},
$$


where $\tau_{\mathrm{a}}$ is the characteristic time of absorption. It is worth to note that the time $\tau_{\mathrm{e}}$ can be comprised as the equilibrium absorption-desorption characteristic time, which is here related to both absorption and desorption coefficients, calculated under equilibrium conditions.

Let us now introduce the gas relaxation time as following:

$$
t_{\mathrm{r}}=\frac{\lambda}{v_{\mathrm{m}}}
$$

where $\lambda$ is the molecular mean free path, which is calculated in the frame of the Hard Sphere (HS) model [10] according to:

$$
\lambda=\frac{\sqrt{\pi}}{2} \frac{\mu}{p} \sqrt{\mathcal{R} T}
$$

In previous equation, $\mu$ is the gas viscosity and $\mathcal{R}$ is the specific gas constant, $\mathcal{R}=k_{\mathrm{B}} / m$. The characteristic speed in Eq. (18) is calculated from:

$$
v_{\mathrm{m}}=\sqrt{\mathcal{R} T},
$$

It is to be noted that the gas relaxation time can be associated to the time between the molecular collisions, or mean free time, which is inverse of the collision frequency.

By analyzing expression (15), one can easily see when the deviation from the Langmuir isotherm is important to be taken into account. We have to compare the gas relaxation time, $t_{\mathrm{r}}$, which characterizes the motion of the gas molecules, with the characteristic equilibrium absorption-desorption time, $\tau_{\mathrm{e}}$, which characterizes the absorption-desorption phenomenon. The following cases can be distinguished:

(i) $t_{\mathrm{r}} \gg \tau_{\mathrm{e}}$, i.e. the flow characteristic time is much larger that the absorption-desorption characteristic time $\tau_{\mathrm{e}}$, therefore absorption-desorption dynamics instantaneously follows the gas dynamics. The surface coverage $\theta(t)$ can be computed by requiring that $R_{a d s}=R_{d e s}$, but replacing Eq. (1) by its non-equilibrium version to compute $R_{a d s}$, Eq. (12), thus:

$$
\theta(t)=\frac{K_{L}^{\prime} J_{m}(t)}{1+K_{L}^{\prime} J_{m}(t)}, \quad K_{L}^{\prime}=\sigma A \tau
$$

(ii) $t_{\mathrm{r}} \ll \tau_{\mathrm{e}}$; in this case the flow characteristic time is too short to influence the absorption-desorption process;

(iii) $t_{\mathrm{r}} \sim \tau_{\mathrm{e}}$, i.e. the flow characteristic time is of the same order as the Langmuir characteristic time $\tau_{\mathrm{e}}$, so the non-equilibrium effects have to be taken into account.

\section{Kinetic model formulation}

In previous Section, the classical Langmuir theory has been briefly described and its limitations have been underlined. This Section is devoted to formulating the absorption-desorption model, based on the kinetic theory of gases. At variance with Langmuir theory, the present one keeps its validity when the gas flow is out of equilibrium but reduces to the classical one when the gas is close to local or global equilibrium.

Let us consider the one-dimensional flow of a gas between two parallel infinite surfaces, fixed at positions $x=0$ and $x=L$, see Fig. 1 . One of the surfaces, located at $x=0$, and kept at the constant and uniform temperature $T_{\mathrm{w} 1}$, can adsorb and desorb the molecules with a probability $\sigma$ and reflect them diffusively with the probability $\alpha_{1}$. The second surface, maintained at the constant and uniform temperature $T_{\mathrm{w} 2}$, 
reflects only the molecules with the probability $\alpha_{2}$ of diffuse reflection. The gas motion in the positive halfspace is governed by the spatially one-dimensional Boltzmann equation, for a polyatomic gas composed of classical rigid rotators as formulated in Ref. [11]:

$$
\frac{\partial f}{\partial t}+v_{x} \frac{\partial f}{\partial x}=\int\left(f\left(\mathbf{v}_{l}^{\prime}, \mathcal{E}_{l}^{\prime} \mid x\right) f\left(\mathbf{v}^{\prime}, \mathcal{E}^{\prime} \mid x\right)-f\left(\mathbf{v}_{l}, \mathcal{E}_{l} \mid x\right) f(\mathbf{v}, \mathcal{E} \mid x)\right) \mathcal{Q} \mathcal{E}_{l}^{\mu} d^{3} v_{l} \mathcal{E}_{l},
$$

where $f(\mathbf{v}, \mathcal{E} \mid x, t)$ is the distribution function of molecular velocity $\mathbf{v}$ and rotational energy $\mathcal{E}$ at location $x$ and time $t$. The exponent $\mu$ takes the values 0 for $j=2$ and 0.5 for $j=3$. In Eq.(22), $\mathcal{Q}$ is defined as:

$$
\mathcal{Q}=\int_{S} d^{2} \hat{e}^{\prime} \int_{0}^{E-\mathcal{E}^{\prime}} \mathcal{E}^{\prime \mu} d \mathcal{E}^{\prime} \times \int_{0}^{E-\mathcal{E}^{\prime}} \mathcal{E}_{l}^{\prime \mu} d \mathcal{E}_{l}^{\prime} \frac{v_{r}^{\prime 2}}{v_{r}} \sigma\left(E ; \hat{\mathbf{e}}^{\prime} \circ \hat{\mathbf{e}} ; \mathcal{E}, \mathcal{E}_{l}^{\prime} \rightarrow \mathcal{E}, \mathcal{E}_{l}\right)
$$

where $\sigma\left(E ; \hat{\mathbf{e}}^{\prime} \circ \hat{\mathbf{e}} ; \mathcal{E}, \mathcal{E}_{l}^{\prime} \rightarrow \mathcal{E}, \mathcal{E}_{l}\right)$ is the differential cross section associated with a binary collision which produces a pair of molecules in the final states $(\mathbf{v}, \mathcal{E}),\left(\mathbf{v}_{l}, \mathcal{E}_{l}\right)$ from a pair of molecules in the initial states $\left(\mathbf{v}^{\prime}, \mathcal{E}^{\prime}\right),\left(\mathbf{v}_{l}^{\prime}, \mathcal{E}_{l}^{\prime}\right)$. The argument $E$ denotes the conserved total energy in the center-of-mass reference frame,

$$
E=\frac{1}{4} m v_{r}^{2}+\mathcal{E}+\mathcal{E}_{l}=\frac{1}{4} m v_{r}^{\prime 2}+\mathcal{E}^{\prime}+\mathcal{E}_{l}^{\prime}
$$

The unit vectors $\hat{\mathbf{e}}^{\prime}=\mathbf{v}_{r}^{\prime} / v_{r}^{\prime}$ and $\hat{\mathbf{e}}=\mathbf{v}_{r} / v_{r}$ have the directions of the relative velocities $\mathbf{v}_{r}^{\prime}=\mathbf{v}_{l}^{\prime}-\mathbf{v}^{\prime}$ and $\mathbf{v}_{r}=\mathbf{v}_{l}-\mathbf{v}$ before and after a collision, respectively. The above model contains the well known Borgnakke-Larsen model (BL) [? ] as a particular case. The latter model is adopted in the present paper to describe binary collision dynamics, accounted by the collision integral, right-hand side of Eq. (22). In the particular version of the BL model considered here, the total collision cross-section is computed from the Hard Sphere potential and a temperature independent rotational relaxation parameter is assumed. The choice of the collision model is adequate for problems like the one discussed here, where temperature variations, within the flow field, are not large.

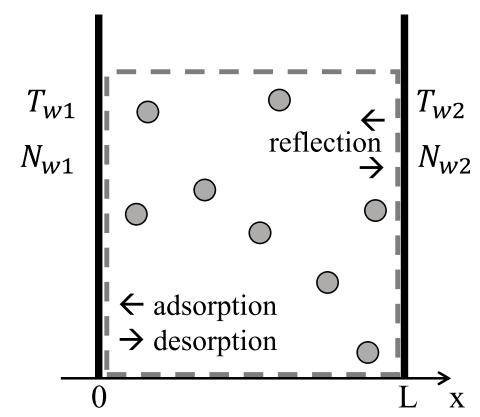

Figure 1: Gas flow between two infinite parallel surfaces, where the left surface is adsorbing-desorbing and right surface is only reflecting.

\subsection{Boundary Conditions}

The boundary condition at the absorbing-desorbing wall defines the molecular flux $J(\mathbf{v}, \mathcal{E} \mid t)$ of the molecules outgoing from the solid surface and injected into the gas region, in terms of the impinging molecular flux. The outgoing flux is composed of two contributions. The first one, $J_{\text {des }}(\mathbf{v}, \mathcal{E} \mid t)$, consists of desorbed molecules whereas the second one, $J_{s c}(\mathbf{v}, \mathcal{E} \mid t)$, consists of gas molecules impinging on the solid surface and instantaneously scattered back to the gas region, i.e. after a residence time on the solid surface, assumed to be much shorter than the absorption time and the mean free time. 
The desorption flux is evaluated accordingly to the following expression:

$$
J_{d e s}(\mathbf{v}, \mathcal{E} \mid t)=\tilde{J}_{d e s}(t) \sqrt{\frac{2 \pi}{\mathcal{R} T_{w 1}}} v_{x} \phi_{w 1}(\mathbf{v}, \mathcal{E})
$$

and the following assumptions are considered:

- The overall desorbed molecules flux $\tilde{J}_{d e s}(t)=\int J_{d e s} d \mathbf{v} d \mathcal{E}$ is proportional to the surface density of the adsorbate:

$$
\tilde{J}_{\text {des }}=\frac{\theta(t)}{\tau} N_{\max }
$$

where $\theta(t)$ and $N_{\max }$ are the fractional coverage and the surface density of free sites of the clean surface, defined in Section 2, and $\tau$ is the residence time of a molecule adsorbed on the surface, defined by Eq. (5).

- Desorbed molecules have the following Maxwellian distribution function:

$$
\phi_{w 1}(\mathbf{v}, \mathcal{E})=\frac{1}{\left(2 \pi \mathcal{R} T_{w 1}\right)^{3 / 2}} \exp \left(-\frac{\mathbf{v}^{2}}{2 \mathcal{R} T_{w 1}}\right) \times \frac{\mathcal{E}^{j / 2-1}}{\Gamma(j / 2)\left(k_{B} T_{w 1}\right)^{j / 2}} \exp \left(-\frac{\mathcal{E}}{k_{B} T_{w 1}}\right), \quad v_{x}>0
$$

being $\Gamma$ the complete Gamma function [7] and $j$ the number of rotational degrees of freedom. Here we consider $j=3$, that is, polyatomic gases with 3 internal degrees of freedom.

The form of the second contribution can be readily obtained from the classical Maxwell gas-surface interaction model, modified to take into account the absorption. In general, let $K_{s c}\left(\mathbf{v}^{\prime}, \mathcal{E}^{\prime} \rightarrow \mathbf{v}, \mathcal{E}\right)$ be the scattering kernel which assigns the probability density that a molecule hitting the solid surface with velocity $\mathbf{v}^{\prime}$ and rotational energy $\mathcal{E}^{\prime}$ is scattered with velocity $\mathbf{v}$ and rotational energy $\mathcal{E}$. Then, $J_{s c}(\mathbf{v}, \mathcal{E} \mid t)$ can be written as:

$$
J_{s c}(\mathbf{v}, \mathcal{E} \mid t)=[1-(1-\theta(t)) \sigma] \int_{v_{x}^{\prime}<0} K_{s c}\left(\mathbf{v}^{\prime}, \mathcal{E}^{\prime} \rightarrow \mathbf{v}, \mathcal{E}\right)\left|v_{x}^{\prime}\right| f\left(\mathbf{v}^{\prime}, \mathcal{E}^{\prime} \mid 0, t\right) d \mathbf{v}^{\prime} d \mathcal{E}^{\prime} .
$$

The previous equation has been derived by assuming that molecules impinging on the clean fraction of the solid surface are adsorbed with constant probability $\sigma$ or scattered back to the gas region with probability $(1-\sigma)$; gas molecules hitting the covered area fraction are always scattered. Moreover, it is assumed that the scattering kernel is the same for the covered and clean surface, to keep the model as simple as possible at this stage of the investigation. Further simplifications can be made by adopting the classical Maxwell gas-surface interaction model [10] to obtain $K_{s c}$ in the form:

$$
\begin{aligned}
K_{s c}\left(\mathbf{v}^{\prime}, \mathcal{E}^{\prime} \rightarrow \mathbf{v}, \mathcal{E}\right)= & \left(1-\alpha_{1}\right) \delta\left(v_{x}+v_{x}^{\prime}\right) \delta\left(v_{y}^{\prime}-v_{y}\right) \delta\left(v_{z}^{\prime}-v_{z}\right) \delta\left(\mathcal{E}^{\prime}-\mathcal{E}\right)+ \\
& \alpha_{1} v_{x} \sqrt{\frac{2 \pi}{\mathcal{R} T_{w 1}}} \phi_{w 1}(\mathbf{v}, \mathcal{E}) .
\end{aligned}
$$

In Eq. (29), $\alpha_{1}$ is the surface accommodation coefficient which tunes the amplitudes of complete specular scattering (both for velocity and rotational energy) and complete diffuse scattering according to the wall Maxwellian $\phi_{w 1}(\mathbf{v}, \mathcal{E})$.

In view of the applications described below, it is convenient to revisit the derivation of Eq. (13) in the light of the boundary condition developed above. Using the fluxes defined above, the rate of change of the number of adsorbed molecules per unit area can be written as:

$$
\frac{d N}{d t}=\tilde{J}_{a d s}-\tilde{J}_{d e s}
$$


where the flux of adsorbed molecules is equal to:

$$
\tilde{J}_{a d s}=(1-\theta(t)) \sigma \int_{v_{x}^{\prime}<0}\left|v_{x}^{\prime}\right| f\left(\mathbf{v}^{\prime}, \mathcal{E}^{\prime} \mid 0, t\right) d \mathbf{v}^{\prime} d \mathcal{E}^{\prime} .
$$

The integral at the right-hand side of Eq. (31) gives the overall impinging molecular flux which determines the absorption rate $\tilde{J}_{a d s}$ upon multiplication by the absorption probability $(1-\theta(t)) \sigma$. It is to be noted that, out of equilibrium, $\tilde{J}_{a d s}$ is a function of $f$, hence its determination requires that Eq. (30) is coupled to the Boltzmann equation (22). Maxwell's gas surface interaction model is adopted to prescribe the boundary condition at the impermeable surface, located at $x=L$ (see Figure 1). Accordingly, it is assumed that:

$$
f(L, \mathbf{v}, \mathcal{E})=\left(1-\alpha_{2}\right) f\left(L,-v_{x}, v_{y}, v_{z}, \mathcal{E}\right)+\alpha_{2} n_{w 2} \phi_{w 2}, \quad v_{x}<0
$$

where $\alpha_{2}$ is the accommodation coefficient at the reflecting surface and $\phi_{w 2}$ has the following form:

$$
\phi_{w 2}(\mathbf{v}, \mathcal{E})=\frac{1}{\left(2 \pi \mathcal{R} T_{w 2}\right)^{3 / 2}} \exp \left(-\frac{\mathbf{v}^{2}}{2 \mathcal{R} T_{w 2}}\right) \times \frac{\mathcal{E}^{j / 2-1}}{\Gamma(j / 2)\left(k_{B} T_{w 2}\right)^{j / 2}} \exp \left(-\frac{\mathcal{E}}{k_{B} T_{w 2}}\right), \quad v_{x}<0
$$

The number density, $n_{w 2}$, is calculated from the classical non-permeability condition

$$
n_{w 2} \sqrt{\frac{R T_{w 2}}{2 \pi}}=-\int_{v_{x}<0} v_{x} f(L, \mathbf{v}, \mathcal{E}) d \mathbf{v} d \mathcal{E} .
$$

It is worth noting that, the boundary condition described above, as already stressed in Refs. [1, 2], has strong similarities with boundary conditions used in modeling evaporation/condensation flows [12], the main difference being represented by the necessity of introducing $\theta$ and its time evolution into the present model.

\section{NUMERICAL TECHNIQUE}

\subsection{Dimensionless Properties}

All the parameters used in the numerical code are presented in their dimensionless form, since normalized results allow a clearer comparison when one single input parameter is swept. The normalization of number density, temperature, distance, surface area and number of binding sites per unit of area (surface number density) have been done in the following way:

$$
n^{*}=\frac{n}{n_{0}}, \quad T^{*}=\frac{T}{T_{w 1}}, \quad x^{*}=\frac{x}{\lambda_{0}}, \quad A^{*}=\frac{A}{\lambda_{0}^{2}}, \quad N^{*}=\frac{N}{\lambda_{0} n_{0}} .
$$

In previous expression we use $n_{0}$, the averaged number density in the gap, $n_{0}=\frac{1}{L} \int_{0}^{L} n d x$, as a reference number density. The Hard Sphere model expression, Eq. (19), [7] is used to evaluate the molecular mean free path $\lambda_{0}$ with $n=n_{0}$. The molecular and macroscopic velocities are normalized using the characteristic velocity, Eq. (20), calculated at the adsorbed wall temperature, $v_{\mathrm{w} 1}=\sqrt{\mathcal{R} T_{\mathrm{w} 1}}$. The time is normalized by the gas relaxation time, Eq. (18) with $\lambda_{0}$ and $v_{\mathrm{w} 1}$ :

$$
t_{\mathrm{r}}=\frac{\lambda_{0}}{v_{\mathrm{w} 1}}, \quad t^{*}=\frac{t}{t_{\mathrm{r}}}
$$


It should be noticed that the reference time $t_{\mathrm{r}}$ in Eq. (36) is the characteristic time between the molecular collisions. The molecular flux, energy, heat of absorption and gas density are normalized according to:

$$
J^{*}=\frac{J}{n_{0} v_{\mathrm{w} 1}}, \quad \mathcal{E}^{*}=\frac{\mathcal{E}}{k_{\mathrm{B}} T_{w 1}}, \quad Q^{*}=\frac{Q}{\mathcal{R}_{g} T_{w 1}}, \quad \rho^{*}=\frac{\rho}{\rho_{0}},
$$

where $\rho_{0}=m n_{0}$.

Equation (13) represents the variation of the surface number density as the difference between fluxes of adsorbed and desorbed molecules. However, in this equation both absorption and desorption coefficients are calculated under the equilibrium flow conditions. Therefore, we propose to use the analogous expression, Eq. (30), which relates the variation of the surface number density to the fluxes of molecules

going to and coming from the adsorbing surface. To obtain the surface coverage from Eq. (30) we can normalize it by $N_{\max }$ and we obtain:

$$
\frac{d \theta(t)}{d t}=(1-\theta) \sigma \frac{J_{i n c}}{N_{\max }}-\frac{\theta}{\tau}
$$

where $J_{\text {inc }}$ is the incident flux

$$
J_{i n c}=\int_{v_{x}^{\prime}<0}\left|v_{x}^{\prime}\right| f\left(\mathbf{v}^{\prime}, \mathcal{E}^{\prime} \mid 0, t\right) d \mathbf{v}^{\prime} d \mathcal{E}^{\prime}
$$

to be computed from the distribution function which is not necessarily coincident with (or close to) the Maxwellian equilibrium.

\subsection{Evolution of Fractional Coverage}

As it was mentioned in Section 2.2, two time scales can be defined for considered problem: the gas flow scale, characterized by the gas relaxation time, $t_{\mathrm{r}}$, Eq. (36), and the absorption-desorption time scale, characterized by absorption equilibrium time, $\tau_{\mathrm{e}}$, Eq. (16). We intend to consider the case (iii), see Section 2.2, when $t_{\mathrm{r}} \sim \tau_{\mathrm{e}}$. Therefore, the evolution of the fractional coverage in time is calculated taking into account the transient flow stage. The value of fractional coverage at time $t^{n+1}$ is calculated by using the corresponding value from previous time step $t^{n}$ and the variation of the surface coverage between the two time steps, as

$$
\theta\left(t^{n+1}\right)=\theta\left(t^{n}\right)+\Delta \theta
$$

where the variation of surface coverage, $\Delta \theta$, is calculated according to the difference of absorption and desorption fluxes:

$$
\Delta \theta=\left(\left(1-\theta\left(t^{n}\right)\right) \sigma \frac{J_{\text {inc }}\left(t^{n}\right)}{N_{\max }}-\frac{\theta\left(t^{n}\right)}{\tau}\right) \Delta t_{a}
$$

where $\Delta t_{a}$ is the absorption-desorption time step. Previous expression corresponds to the numerical approximation of the fluxes balance equation (38). To solve together Eq. (22) by the DSMC technique and Eqs. (40), (41), we need to adapt the time step usually used in the DSMC to the time scale of the absorption-desorption phenomena, which can be very different. Further details on the choice of the numerical parameter are given in next Section.

The proposed technique which allows the surface coverage to be determined by the balance of fluxes, Eq. (38), is called in the following text as the "fluxes method". This method will be compared to the results obtained from Langmuir isotherm. This means that after the calculation of the surface coverage by 
the fluxes balance equation, Eqs. (40) and (41), the fractional coverage is also calculated from Langmuir isotherm expression:

$$
\theta\left(t^{n+1}\right)=\frac{K_{L} p\left(t^{n+1}\right)}{1+K_{L} p\left(t^{n+1}\right)}
$$

where the $K_{L}$ coefficient, Eq. (10), is constant when the surface temperature is kept constant.

\subsection{Numerical Procedure and Simulation Parameters}

The spatial one-dimensional kinetic equation (22) is solved using the DSMC method. The developed DSMC code, here extended to the absorption-desorption boundary condition, had previously validated against deterministic solutions of a kinetic model equation for polyatomic gases in Ref. [13]. The simulation of the molecular collisions has been done with the classical NTC model [7, 14]. To start the simulations, firstly, the initial state is set and the initial position and velocity are defined for all the simulated particles. After that, all the particles are moved according to their respective velocities, and the interactions between particles and boundaries are evaluated. In the the next time step, the particles are set inside the new cells and, again, the binary collisions between the particles inside each cell and the interactions with the boundaries are carried out. The calculations stop when the steady regime is achieved. Besides, the macroscopic flow properties at steady regime and the evolution of some parameters in time are calculated.

Initially, several test cases have been carried out to check the convergence of numerical method and find the optimal set of computational grid parameters. The following parameters have been varied: the initial number of particles $\mathcal{N}_{0}$, the cells size $\Delta x$, the DSMC time step $\Delta t$, the absorption-desorption time step $\Delta t_{a}$ and the maximum time of simulation $t_{\max }$. After analyzing the influence of these input parameters on the gas macroscopic quantities and fractional coverage for several rarefaction levels, their optimal sets were found, see Table 1, and then fixed for all the simulations presented in this work. It should be mentioned that all the parameters shown in this table are presented in their dimensionless form.

\begin{tabular}{lc|ccccc}
\hline Input parameter & Var. & $\mathrm{Kn}=0.01$ & $\mathrm{Kn}=0.1$ & $\mathrm{Kn}=1$ & $\mathrm{Kn}=10$ & $\mathrm{Kn}=\infty$ \\
\hline length of domain & $x_{\text {max }}^{*}$ & 100 & 10 & 1 & 0.1 & 0.001 \\
number of cells & $N_{\Delta x}$ & 500 & 100 & 100 & 100 & 100 \\
size of each cell & $\Delta x^{*}$ & 0.2 & 0.1 & 0.01 & 0.001 & $10^{-5}$ \\
number of particles & $\mathcal{N}_{0} \times 10^{-3}$ & 50 & 50 & 50 & 50 & 10 \\
particles per cell & $\mathcal{N}_{0, c}$ & 100 & 500 & 500 & 500 & 100 \\
DSMC time step & $\Delta t^{*}$ & 0.001 & 0.001 & 0.001 & $10^{-4}$ & $10^{-6}$ \\
average number of time steps & $n_{a v} \times 10^{-3}$ & 1 & 1 & 1 & 10 & 1 \\
absorption time step & $\Delta t_{a}^{*}$ & 1 & 1 & 1 & 1 & 0.001 \\
simulation time & $t_{\max }^{*}$ & 5000 & 1000 & 1000 & 50 & 5 \\
\hline
\end{tabular}

Table 1: Dimensionless input parameters used in all the simulations.

The rarefaction level of the flow is a function of the Knudsen number, defined as the ratio between the molecular mean free path and the characteristic flow dimension, here the distance between plates, i.e. $K n=\lambda_{0} / L$.

As it can be seen in Table 1, two different time steps are used in the calculations. The first one is the DSMC time step, $\Delta t$, during which the position and velocity of the particles are updated, and the binary collision between particles and interaction between particles and boundaries are evaluated. The second one is the absorption time step, $\Delta t_{a}$, used to update the fractional coverage, and it is related to the DSMC time step in the following way:

$$
\Delta t_{a}=n_{a v} \Delta t
$$


where $n_{a v}$ is the average number of DSMC time steps used to evaluate the macroscopic properties in the DSMC code. Both of these time steps should be small enough to guarantee the numerical convergence.

To start the simulation, the initial value of fractional coverage has to be set equal to an arbitrary value between 0 to 1 or calculated from the Langmuir absorption isotherm, Eq. (8), for $p=n_{w 1} k_{B} T_{w 1}$. The last choice of the initial fractional coverage leads to faster convergence, but the final value of fractional coverage in steady state regime is independent of this initial value, which will be shown in next Section.

The absorption and desorption phenomena for a pair gas-surface is characterized by the heat of absorption, the residence time constant and the gas and surface temperatures. In this study we do not focus on the study of a given pair of a gas and a surface, but we simply analyze the influence of the governing parameters on the absorption-desorption process. To do this, we consider only dimensionless variables without referring to a specific pair of a gas and a surface, then we will vary them in a reasonable way to analyze qualitatively their influence on the absorption-desorption rate and surface coverage. However, to have an idea about the order of magnitude of the possible dimensional parameters a generic case presented in Ref. [4] is used for an illustration.

The authors of Ref. [4] showed that, for Nitrogen adsorbing in Ruthenium at $T=300 K$, the heat of absorption is $Q=31 \mathrm{kJmol}^{-1}$ and the residence time constant is $\tau_{0}=10^{-13} \mathrm{~s}$. To obtain the dimensionless values of these parameters, using Eqs. (36) and (37), we fixed the gas temperature to be $300 \mathrm{~K}$ and the atmospheric pressure, $p_{0}=1.01325 \times 10^{5} \mathrm{~Pa}$, so the corresponding number density is equal to $n_{0}=$ $2.45 \times 10^{25} \mathrm{~m}^{-3}$. Considering again Nitrogen with the Hard Sphere molecular diameter $d=3.76 \times 10^{-10} \mathrm{~m}$ we obtain the molecular mean free path, using Eq. (19), equal to $6.52 \times 10^{-8} \mathrm{~m}$. By fixing the adsorbing (reference) surface temperature to be equal to $T_{\mathrm{w} 1}=300 \mathrm{~K}$, the gas reference time becomes equal to $t_{\mathrm{r}}=$ $2.19 \times 10^{-10} \mathrm{~s}$. Considering these dimensional parameters, the dimensionless values of heat of absorption and residence time constant are equal to $Q^{*}=12.4$ and $\tau_{0}^{*}=4.57 \times 10^{-4}$, respectively. The surface area of one binding site can be calculated as $A=\pi d^{2} / 4$, which for the Hard Sphere diameter of Nitrogen gives $A=1.11 \times 10^{-19} \mathrm{~m}^{2}$. Finally, we can also estimate the maximal number of molecules which can be adsorbed at bare surface per unit of area, from the inverse of the surface area of one binding site, i.e. $N_{\max }=1 / A=9.03 \times 10^{18} \mathrm{~m}^{-2}$. Its dimensionless value reads 5.66 , so fixing it equal to 1 in the numerical code is reasonable. In the code $A^{*}=1$, so dimensional value of $A$ is $4.26 \times 10^{-15} \mathrm{~m}^{2}$.

Finally, the Borgnakke-Larsen model contains an input parameter, called mixing parameter $Z$, which determines the fraction of non-elastic collisions. In all of our analysis, its value was kept constant, being $Z=0.3[15]$.

\section{PARAMETRIC STUDY OF absorption-DESORPTION MODEL}

The present Section is mainly devoted to describing the results of several numerical tests aiming at assessing the influence of some model parameters on the absorption-desorption process, as shown by the behavior of the coverage fraction and the properties of the gas macroscopic quantities.

Before the description of the parametric study results, two groups of test results are presented at first, in subsections 5.1 and 5.2, in order to provide a preliminary self-consistency check of the model. ${ }^{1}$

\subsection{Gas absorption in near equilibrium conditions}

\footnotetext{
${ }^{1}$ All the numerical results are presented in their dimensionless form
} 
Within the simplification of one-dimensional geometry described above, the numerical test described here mimics the experimental technique often adopted to characterize the kinetics of gas absorption on solid surfaces [16]. The latter consists into exposing an initially clean solid surface to a gaseous environment, confined in a vessel. The following gas absorption causes the gas pressure to decrease exponentially till absorption and desorption reach equilibrium. On ordinary spatial scales, the experimental process takes a few minutes, ensuring that evolution takes places as a sequence of equilibrium states.

Accordingly, we simulate, at first, the simple condition when the temperatures of the adsorbing and reflecting surfaces are equal, that is $T_{w 1}=T_{w 2}$. Under this condition, a reference pressure, $p_{\mathrm{e}}$, is fixed and the corresponding Langmuir equilibrium surface coverage, $\theta_{\mathrm{i}}=f\left(p_{\mathrm{e}}, T_{\mathrm{w} 1}\right)$, is computed, according to Eq. (8). Then, the kinetic model is used to simulate the surface coverage evolution with time, which is obtained to be constant and equal to its initial value $\theta_{\mathrm{e}}=\theta_{\mathrm{i}}=0.637$, see solid black line on Fig. 2. To check the influence of the initial conditions we simulated also two limit cases. In the first one, a very low pressure is set in the gap, so low that value of initial surface coverage, $\theta_{\mathrm{i}}$, calculated according to Eq. (8), is negligible. Then, the pressure is changed instantaneously to $p_{\mathrm{e}}$, which was used in previous test case. As it is seen from Fig. 2(orange dashed line), $\theta(t)$ evolves towards its prescribed equilibrium value, $\theta_{\mathrm{e}}=0.637$. The second limit case was simulated by fixing initially the pressure large enough to obtain a fully covered surface, $\theta_{\mathrm{i}}=1$. As in previous case, the pressure was changed instantaneously up to $p_{\mathrm{e}}$. As seen from Fig. 2 (red dashed line), the numerical solution evolves to the same equilibrium state, with $\theta_{\mathrm{e}}=0.637$.

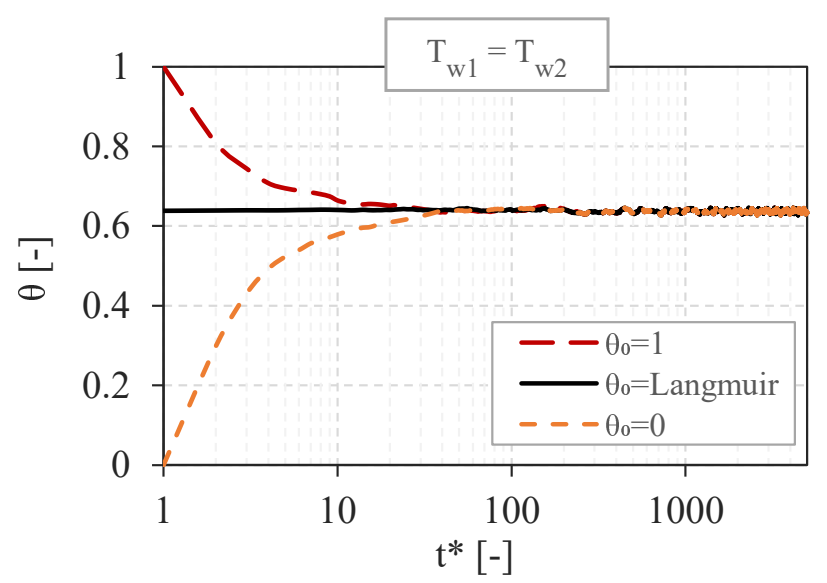

Figure 2: Evolution of fractional coverage obtained by fluxes method for different values of initial fractional coverage $\left(\theta_{\mathrm{i}}\right)$ keeping both surfaces at the same temperature. Simulation parameters: $K n=0.01, T_{w 2} / T_{w 1}=1, \tau_{0}^{*}=2 \cdot 10^{-4}, Q^{*}=10$, $\sigma=1$ and $\alpha_{1}=\alpha_{2}=1$.

\subsection{Gas absorption in non-equilibrium conditions}

The second model test aims at highlighting the effects of gas phase non-equilibrium on absorption/desorption rates. As in the first test, the temperatures of both surfaces were initially set to be equal to $T_{\mathrm{w} 1}$, the initial pressure set equal to $p_{\mathrm{e}}$ and the surface coverage, as calculated from Eq. (8), set equal to $\theta_{\mathrm{e}}=0.637$. Then, the temperature of the reflecting plate was changed instantaneously up to $T_{\mathrm{w} 2}=2 T_{\mathrm{w} 1}$. As a result of the pressure increase, the surface coverage, calculated by the proposed model, starts to varying in time up to reaching a new steady value, higher than the initial equilibrium one, equal to 0.716 (see solid black line on Fig. 3). The obtained steady condition does not depend on initial surface coverage fraction, as shown by the results of simulations in which we changed the initial coverage to be 
respectively equal to 0.0 and 1.0 and we obtained the convergency of the two solutions to the same final value equal to 0.716 (see dashed orange and red lines in Fig. 3. The computed steady value of the surface coverage, which takes into account the temperature gradient in the gas, is in agreement with the value calculated using the Langmuir theory.

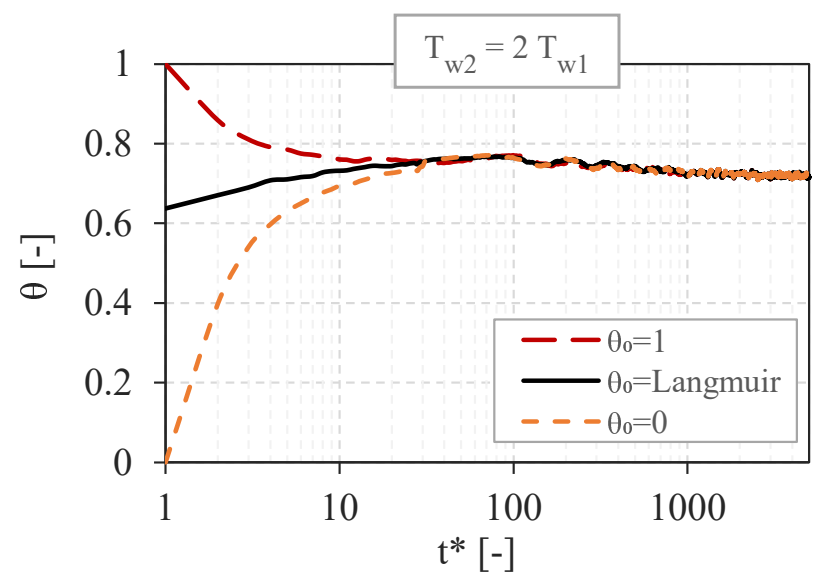

Figure 3: Evolution of fractional coverage obtained by fluxes method for different values of initial fractional coverage $\left(\theta_{0}\right)$. Initial conditions: $K n=0.01, T_{w 2} / T_{w 1}=2, \tau_{0}^{*}=2 \cdot 10^{-4}, Q^{*}=10, \sigma=1$ and $\alpha_{1}=\alpha_{2}=1$.

Actually, for the previous non-equilibrium test cases the Knudsen number value was relatively small, being equal to 0.01. Therefore, deviations from equilibrium are accordingly small and the value of the obtained surface coverage from the kinetic model is very close to Langmuir theory prediction. Figure 4a) provides the comparison between the evolution of the surface coverage, obtained by the kinetic model and Langmuir theory for the case $T_{\mathrm{w} 2}=2 T_{\mathrm{w} 1}$. It is seen that, after a transient regime, both values come to coincide and the final common value of $\theta$ is equal to 0.716 (see also Fig. 3, where the influence of the initial condition was tested for the same conditions)

As it was explained in Section 3 the new model, in contrast to Langmuir model, allows us to simulate both equilibrium and non-equilibrium gas flow conditions. To assess the sensitivity of the absorptiondesorption model the degree of non-equilibrium, as expressed by the Knudsen number, new simulations with $K n=10$, were carried out keeping the same model settings of the non-equilibrium tests described above but reducing the distance $L$ between the plates. As it is clear from Fig. 4b) the evolution of the surface coverage, obtained by the kinetic model for this higher Knudsen number, differs from the previous ones under two main aspects. First, the final steady-state surface coverage is now equal to 0.647, instead of 0.716, as obtained for $K n=0.01$, see Fig. 4a). Second, for $K n=10$, Langmuir theory provides a slightly higher value, 0.686 , since the equilibrium relation between gas phase pressure and impinging surface flux is no longer valid.

\subsection{Evaluation of the Surface Coverage}

In this section we present the parametric study of the influence of the governing parameters of the model on the surface coverage. Along this study, we consider as the reference case the one which has the following parameters: $K n=0.01, T_{\mathrm{w} 2} / T_{\mathrm{w} 1}=2, \tau_{0}^{*}=2 \cdot 10^{-4}, Q^{*}=10, \sigma=1$ and $\alpha_{1}=\alpha_{2}=1$.

We start our study with the comparison of the absorption and desorption fluxes which balance changes the surface coverage, see Eq. (30). Figure 5 shows the evolution of these fluxes with time for two values of the Knudsen number, $K n=0.01$ and 10. Initially, the partial coverage is assumed, $\theta=0.637$. From these graphs, it is clear that in the beginning of simulations the absorption flux is larger than the desorption 


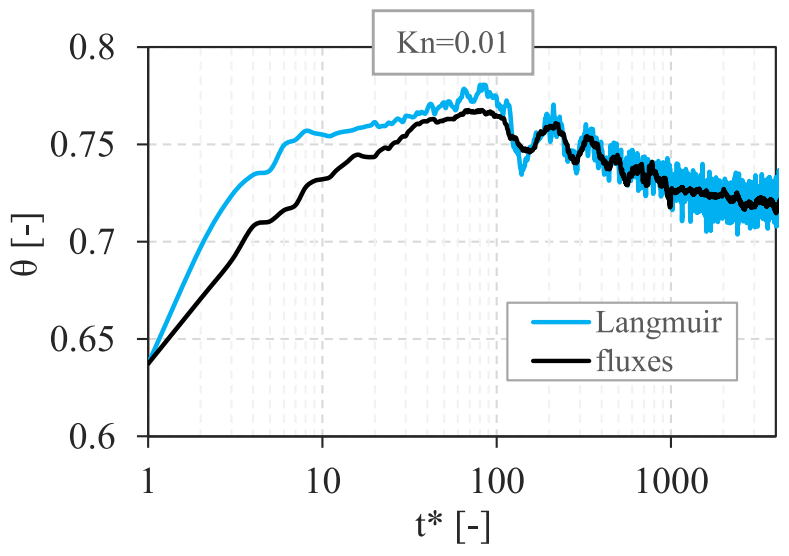

(a)

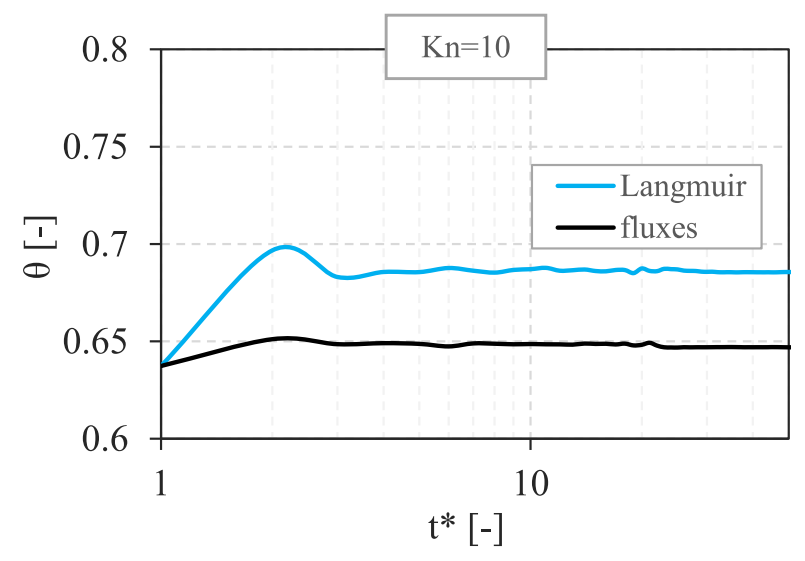

(b)

Figure 4: Evolution of fractional coverage obtained by fluxes method and Langmuir expression for two different values of rarefaction level, (a) $K n=0.01$ and (b) $K n=10$. Initial conditions: $T_{w 2} / T_{w 1}=2, \tau_{0}^{*}=2 \cdot 10^{-4}, Q^{*}=10, \sigma=1$ and $\alpha_{1}=\alpha_{2}=1$.

one, then, after some time period, which can be called relaxation time (or time to reach an equilibrium), both fluxes become equal. This relaxation time is larger for smaller Knudsen number.

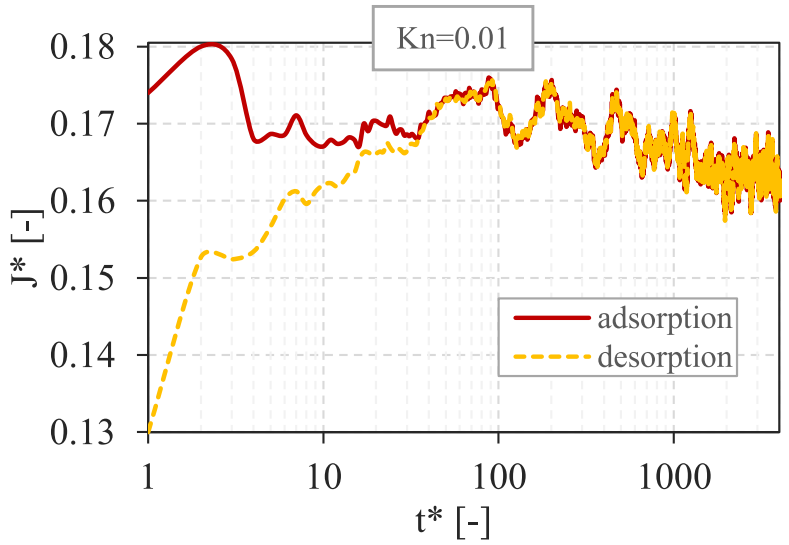

(a)

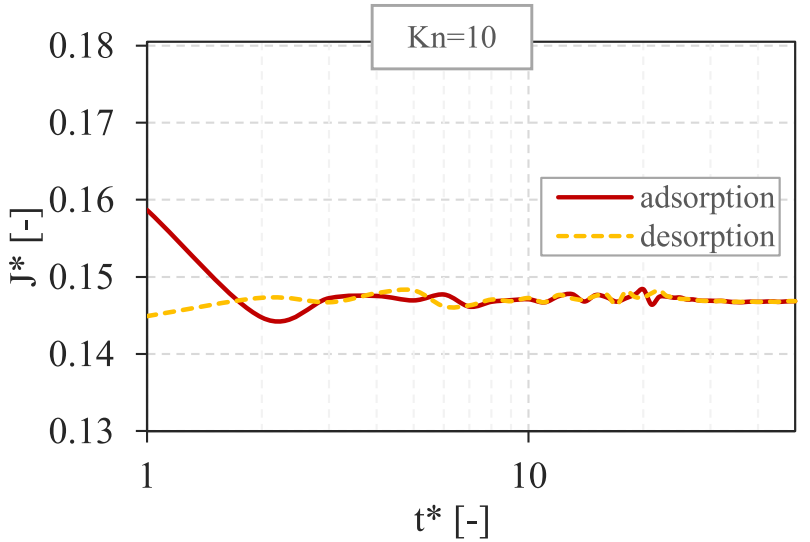

(b)

Figure 5: Evolution of absorption and desorption fluxes for two different flow rarefaction levels: (a) $K n=0.01$ and (b) $K n=10$. Initial conditions: $T_{w 2} / T_{w 1}=2, \tau_{0}^{*}=2 \cdot 10^{-4}, Q^{*}=10, \sigma=1$ and $\alpha_{1}=\alpha_{2}=1$.

Now we quantify the influence of the gas rarefaction, namely of the Knudsen number, on the evolution in time of the surface coverage. Figure 6 presents this evolution of the surface coverage for different rarefaction levels, i.e. Knudsen number, 0.01, 0.1, 1, 10 and $\infty$. The fractional coverage decreases when the rarefaction level increases, as expected, since with the increase of rarefaction level the gas number density decreases also, therefore a lower number of molecules are available to be adsorbed to the surface. The lower is the Knusden number, the slower is the convergence process, i.e. the longer is the time to obtain the steady state solution, $t_{\mathrm{ss}}$. For instance, for $K n=0.01$ the steady regime is reached approximately when $t_{\mathrm{ss}} \approx 3000$, however for $K n=10$, the convergence is achieved when $t_{\mathrm{ss}} \approx 5$. The time needed to 
achieve the steady-state value of the surface coverage, $t_{\mathrm{ss}}$, is provided in Table 2 for five different levels of rarefaction. This time to reach the steady-state surface coverage is larger then the relaxation time, the time needed to equilibrate the absorption and desorption fluxes, see Figure 5. However, the behavior for both times with the increasing of the gas rarefaction is the same: higher is the rarefaction shorter is the time to reach the equilibrium.

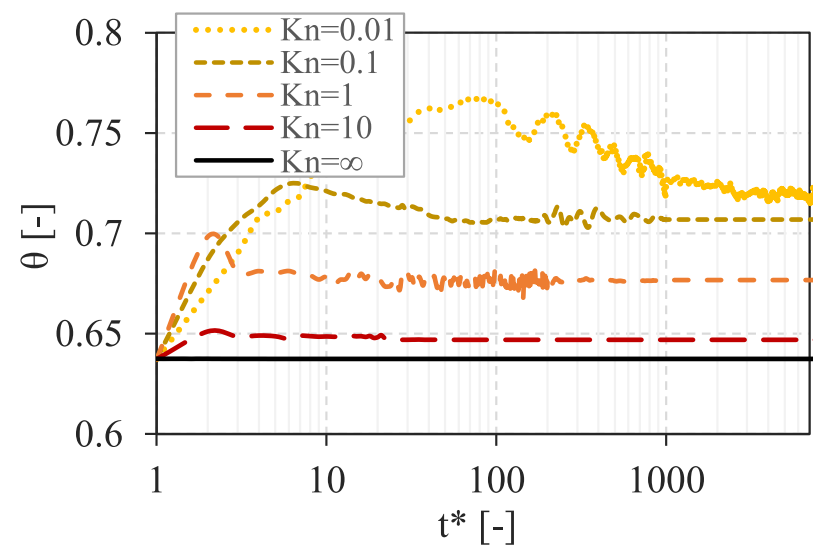

Figure 6: Evolution of fractional coverage obtained by fluxes method for different values of rarefaction level $(K n)$. Initial conditions: $T_{w 2} / T_{w 1}=2, \tau_{0}^{*}=2 \cdot 10^{-4}, Q^{*}=10, \sigma=1$ and $\alpha_{1}=\alpha_{2}=1$,

\begin{tabular}{c|ccccc}
\hline $\mathrm{Kn}$ & 0.01 & 0.1 & 1 & 10 & $\infty$ \\
\hline$t_{\mathrm{ss}}$ & 3000 & 800 & 200 & 30 & $\approx 0$ \\
\hline
\end{tabular}

Table 2: Dimensionless time $t_{\mathrm{ss}}$ needed to achieve the steady-state value of fractional coverage $\theta$.

According to the Langmuir theory, the absorption and desorption phenomena are influenced by several parameters, being two of them the heat of absorption, $Q$, and the residence time constant, $\tau_{0}$, are directly related to the surface nature and the gas hitting the surface.

The influence of the heat of absorption, $Q$, on the fractional coverage is shown in Fig. 7 for two different Knudsen numbers, $K n=0.1$ and 10. As it can be seen in Fig. 7a, when the heat of absorption increases, the value of the fractional coverage increases as well. The higher is the heat of absorption, the more energy is needed for a molecule to be desorbed from a surface. As a consequence of it, the higher is the heat of absorption, the higher is the surface coverage. For the cases considered here, the dimensionless values of heat of absorption were varied from 6 to 12. These dimensionless values of the heat of absorption are considered relatively small and correspond to physisorption, i.e. the associated dimensional value of $Q$ is lower than $100 \mathrm{~kJ} \mathrm{~mol}^{-1}$. Comparing the two graphs from Fig. 7, it can be concluded that the increase in the rarefaction level slightly decreases the surface coverage for the corresponding heat of absorption. For instance, for $Q^{*}=10$, represented by the red long dash line in Fig. 7, the steady-state value of fractional coverage is $\theta=0.707$ when $K n=0.1$ and it decreases to $\theta=0.647$ for $K n=10$, being the difference approximately $9 \%$.

Figure 8 shows that the increase of the residence time constant $\tau_{0}$ implies in the increase of the fractional coverage. Again, raising the residence time constant means to keep the molecules attached at the surface for a longer time, and for this reason, the value of fractional coverage becomes higher. As for the heat of absorption, the increasing in the rarefaction level decreases slightly the surface coverage for the given residence time constant. 


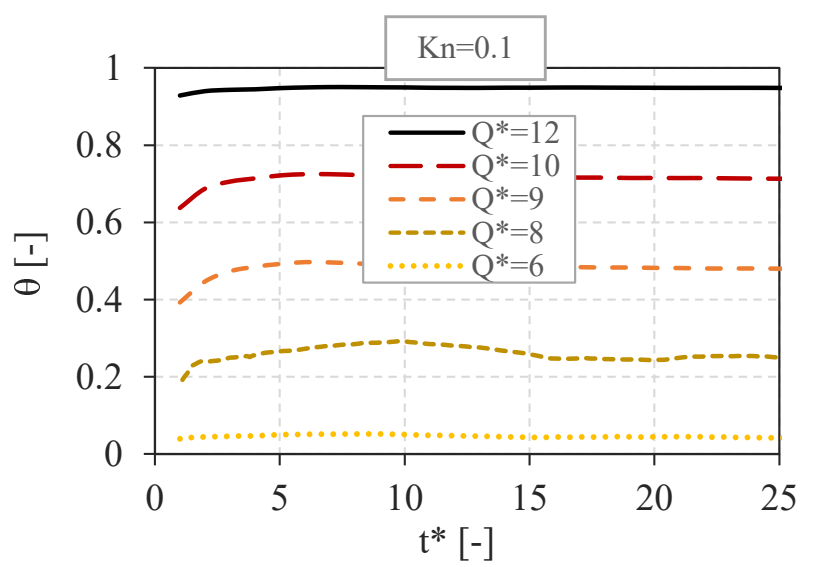

(a)

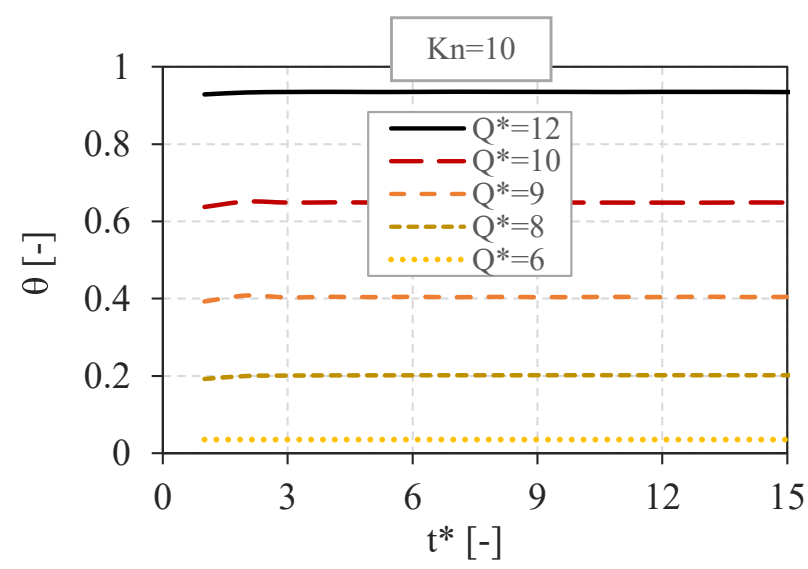

(b)

Figure 7: Evolution of fractional coverage for different values of dimensionless heat of absorption $Q^{*}$ for two different rarefaction levels: (a) $K n=0.1$ and (b) $K n=10$. Initial conditions: $T_{w 2} / T_{w 1}=2, \tau_{0}^{*}=2 \cdot 10^{-4}, \sigma=1$ and $\alpha_{1}=\alpha_{2}=1$.

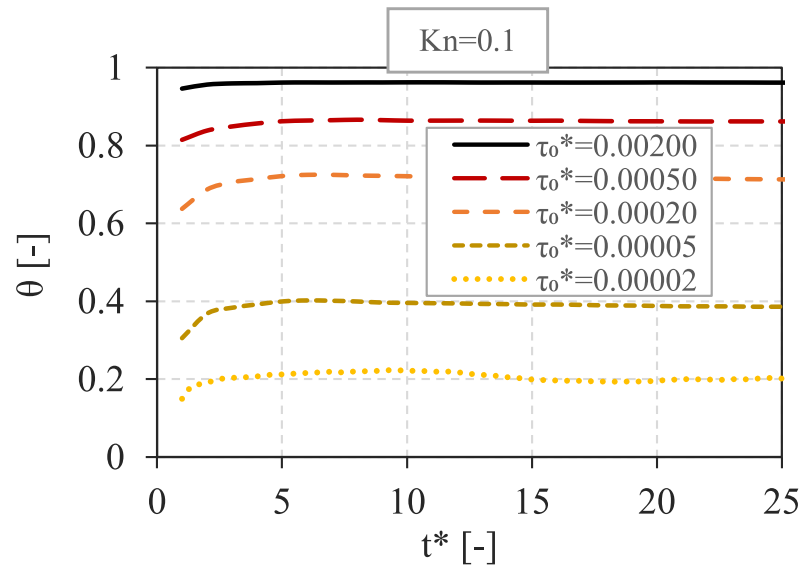

(a)

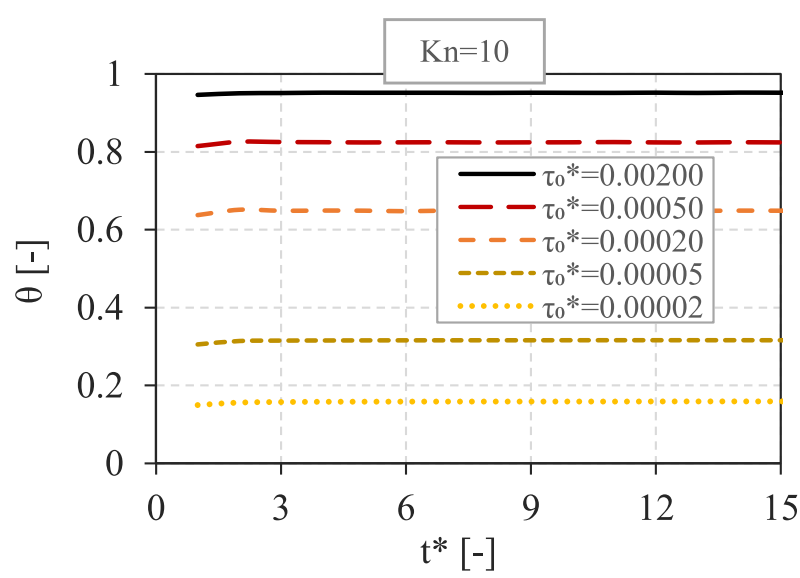

(b)

Figure 8: Evolution of fractional coverage for different values of dimensionless residence time constant $\tau_{0}$ for two different rarefaction levels: (a) $K n=0.1$ and (b) $K n=10$. Initial conditions: $T_{w 2} / T_{w 1}=2, Q^{*}=10, \sigma=1$ and $\alpha_{1}=\alpha_{2}=1$.

The state of the gas near the surface could also impact the surface coverage. When changing the reflecting wall temperature, the temperature in the gap changes also. Figure 9 shows the influence of the reflecting wall temperature, $T_{w 2}$, on the fractional coverage for two different Knudsen numbers, 0.1 and 10. The increasing of the reflecting wall temperature for the fixed rarefaction level leads to pressure increasing in the gap, Fig. 11. As both absorption and desorption coefficients depend only on the adsorbing wall temperature, which is kept constant here, the increasing in pressure leads to increasing of the absorption rate, so to increasing of the surface coverage. Comparing the graphs from Figs. 9a and 9b, it can be concluded also that for low level of rarefaction, the influence of the reflecting wall temperature is much higher than for higher level of rarefaction.

From Figs. 7, 8 and 9, it can be concluded that increasing the dimensionless heat of absorption from 6 to 12 , and also increasing the dimensionless residence time constant from $2 \cdot 10^{-5}$ to $2 \cdot 10^{-3}$ has much more 


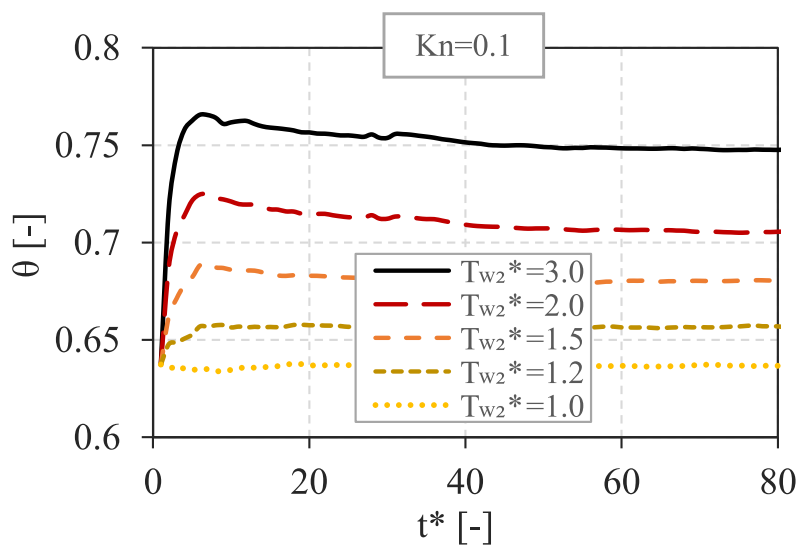

(a)

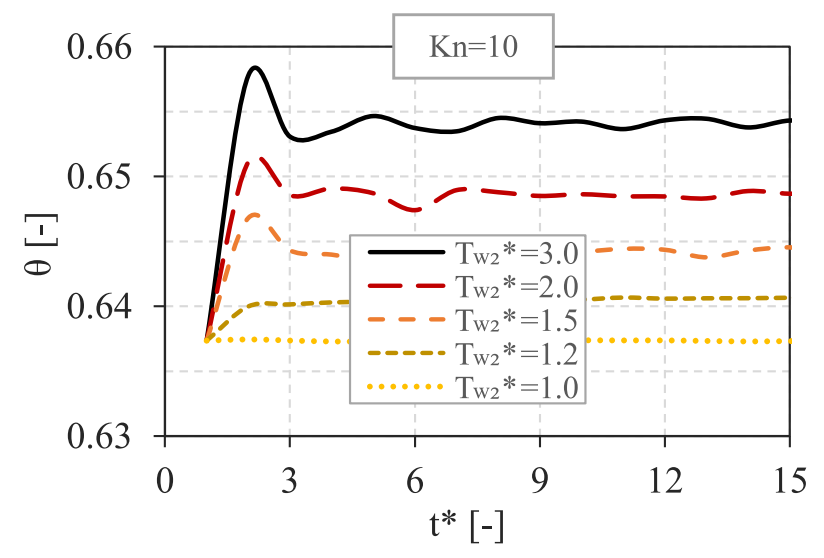

(b)

Figure 9: Evolution of fractional coverage for different values of dimensionless reflecting surface temperature $T_{w 2}^{*}$ for two different rarefaction levels: (a) $K n=0.1$ and (b) $K n=10$. Initial conditions: $\tau_{0}^{*}=2 \cdot 10^{-4}, Q^{*}=10, \sigma=1$ and $\alpha_{1}=\alpha_{2}=1$.

influence on the surface coverage than increasing the dimensionless reflecting wall temperature from 1 to 3 , and therefore increasing the gas temperature near the adsorbing surface, independently of the Knudsen number. It was confirmed that all of these parameters, temperature, heat of absorption and residence time constant play an important role in the absorption and desorption processes.

The last parameter that was varied in this analysis was the absorption probability at the adsorbing surface, $\sigma$. Figure 10 shows the influence of absorption probability on the fractional coverage, $\theta$. As can be seen from this graph, and as it was expected, the higher is the absorption probability, the higher is the fractional coverage. However, the absorption probability equal to one does not lead to the total surface coverage because this value is strongly impacted by the heat of absorption and the residence time constant.

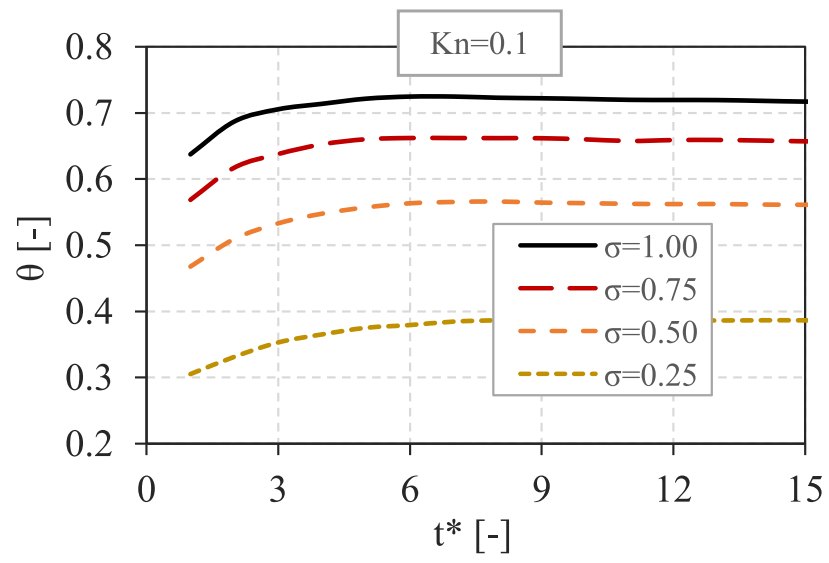

Figure 10: Evolution of fractional coverage for different values of absorption probability $\sigma$. Initial conditions: $K n=0.1$, $T_{w 2} / T_{w 1}=2, \tau_{0}^{*}=2 \cdot 10^{-4}, Q^{*}=10$ and $\alpha_{1}=\alpha_{2}=1$

\subsection{Macroscopic Gas Properties}

Along this section, the steady-state profiles of the gas macroscopic parameters between the two parallel surfaces are presented and analyzed considering different initial conditions All the profiles are plotted as 
a function of the dimensionless distance between two surfaces, $L^{*}$, where $L^{*}=x / L$.

Figure 11 shows the profile of dimensionless pressure between the two surfaces for different values of temperature of the reflecting wall and for two values of the Knudsen number, $K n=0.1$ and 10 . As it can be seen, the pressure in the gas increases when the temperature of the reflecting wall is increased. Higher pressures are obtained for the smaller rarefaction level.

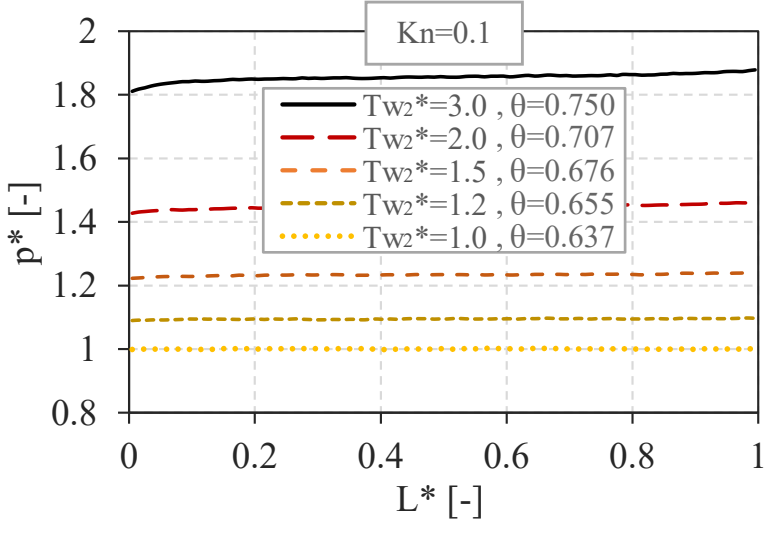

(a)

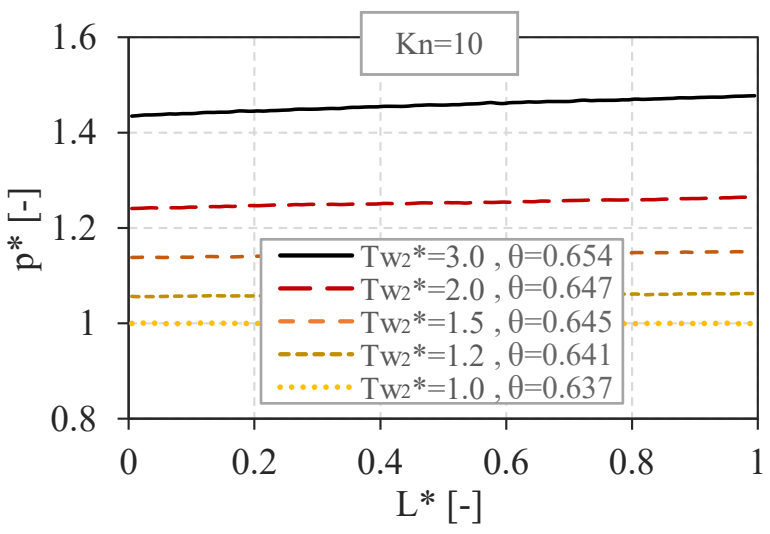

(b)

Figure 11: Dimensionless pressure profiles between the two surfaces for five different values of temperature of reflecting wall $T_{w 2}$. Initial conditions: $\tau_{0}^{*}=2 \cdot 10^{-4}, Q^{*}=10, \sigma=1$ and $\alpha_{1}=\alpha_{2}=1$.

Figure 12 shows the temperature and density profiles between the two surfaces for different levels of rarefaction. Figure 12a demonstrates the impact of the gas rarefaction on the temperature equilibrium near the surface: the higher is the rarefaction level, the larger is the difference between gas and surface temperatures, i.e. temperature jump. However, under considered condition, the presence of the adsorbing wall does not modify the intensity of temperature jump near the surface.

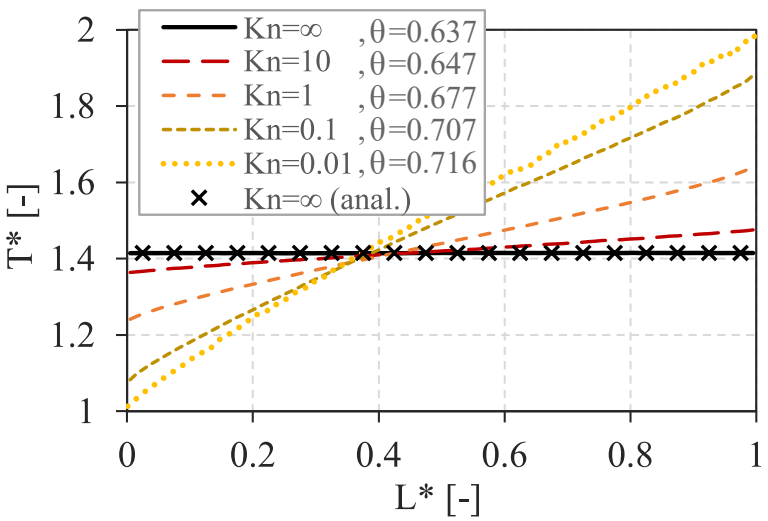

(a)

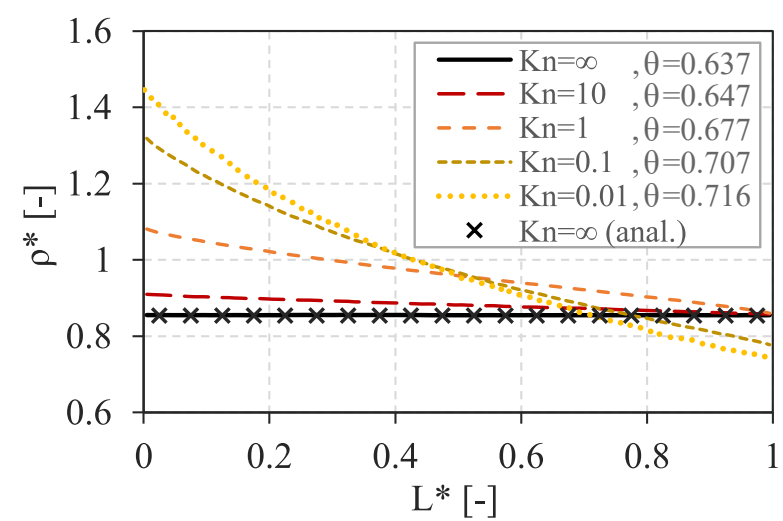

(b)

Figure 12: Dimensionless (a) temperature and (b) density profiles between the two surfaces for five different rarefaction levels $(K n)$. The lines show the results obtained by the numerical code while the analytical solution is represented by the black $\mathbf{x}$ markers. Initial conditions: $T_{w 2} / T_{w 1}=2, \tau_{0}^{*}=2 \cdot 10^{-4}, Q^{*}=10, \sigma=1$ and $\alpha_{1}=\alpha_{2}=1$.

The profiles of the translational, parallel and rotational temperatures for $K n=0.01$ and 10 are 
presented in Fig. 13. The complete equilibrium between all temperatures and between the gas and surface temperatures can be observed on Fig. 13(a). For larger Knudsen number, Fig. 13(b), the temperature jump is visible near the wall, since the gas temperature is $T^{*} \approx 1.37$ and the wall is maintained at $T_{w 1}^{*}=1$. For this relatively large Knudsen number, the equilibrium between the translational, parallel and rotational temperatures starts to disappear, and the parallel temperature becomes different from the other ones.

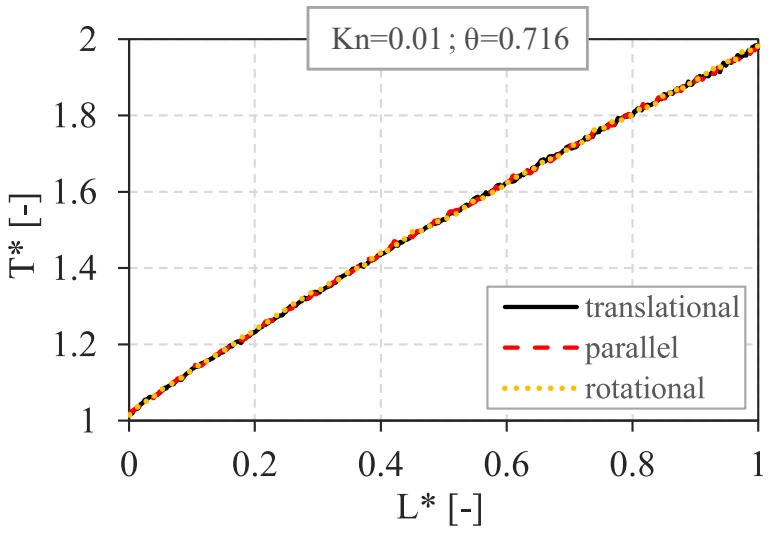

(a)

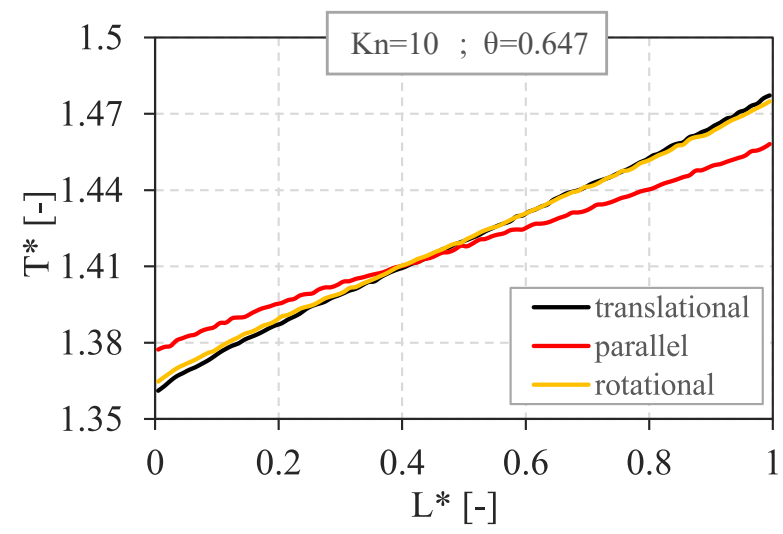

(b)

Figure 13: Profiles of dimensionless translational, parallel and rotational temperatures along the space between the two plates for two rarefaction levels: (a) $K n=0.01$ and (b) $K n=10$. Initial conditions: $T_{w 2} / T_{w 1}=2, \tau_{0}^{*}=2 \cdot 10^{-4}, Q^{*}=10$, $\sigma=1$ and $\alpha_{1}=\alpha_{2}=1$

Now the influence of the accommodation coefficients at the adsorbing wall, $\alpha_{1}$, is analyzed under following conditions: $K n=0.1, T_{w 2} / T_{w 1}=2, \tau_{0}^{*}=2 \cdot 10^{-4}, \sigma=1$ and $\alpha_{2}=1$. Figures 14 and 15 show the temperature and density profiles between the two surfaces for different values of $\alpha_{1}$ and for two heat of absorption, $Q^{*}=10$ and 12, respectively. From both figures, the impact of the changes in the accommodation coefficient at the adsorbing wall, $\alpha_{1}$, is visible and it is more pronounced when the heat of absorption is higher. For this case, $Q^{*}=12$, the number of adsorbed molecules at the surface is higher, diminishing the number of free sites available for absorption (proportional to $1-\theta$ ), Eq. (31), consequently increasing the probability of reflection. It is clear that, increasing the number of reflected molecules, the importance of the accommodation coefficient becomes higher.

Figure 16 shows the influence of the accommodation coefficient at the reflecting surface, $\alpha_{2}$, on the temperature and density profiles. The higher is the accommodation coefficient at this surface, the smaller is the temperature jump near this wall. For instance, for $\alpha_{2}=1$, represented by the black solid line, the value of the gas temperature very close to the reflecting wall is 1.88 , while for $\alpha_{2}=0.1$, represented by the dark yellow short dash line, the gas temperature close to the reflecting wall is approximately 1.28, considerably lower than the reflecting wall temperature, $T_{w 2}^{*}=2$.

\section{CONCLUSION}

A new approach for simulation of non-equilibrium absorption and desorption phenomena is proposed by extending and modifying the classical equilibrium Langmuir absorption model to non-equilibrium condition at the adsorbing boundaries. This approach is based on the numerical solution of the Boltzmann equation and a kinetic boundary condition which dynamically couples the adsorbed/desorbed fluxes to the solid surface coverage. The new absorption-desorption boundary condition is implement in the DSMC method 


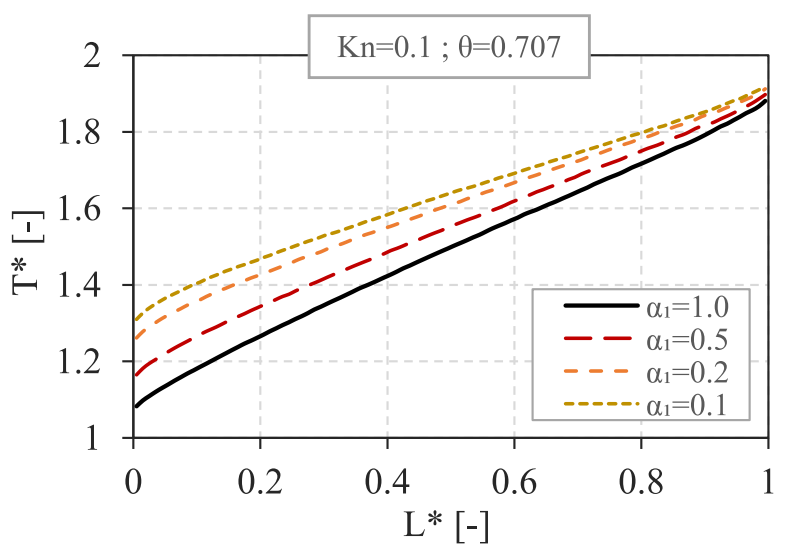

(a)

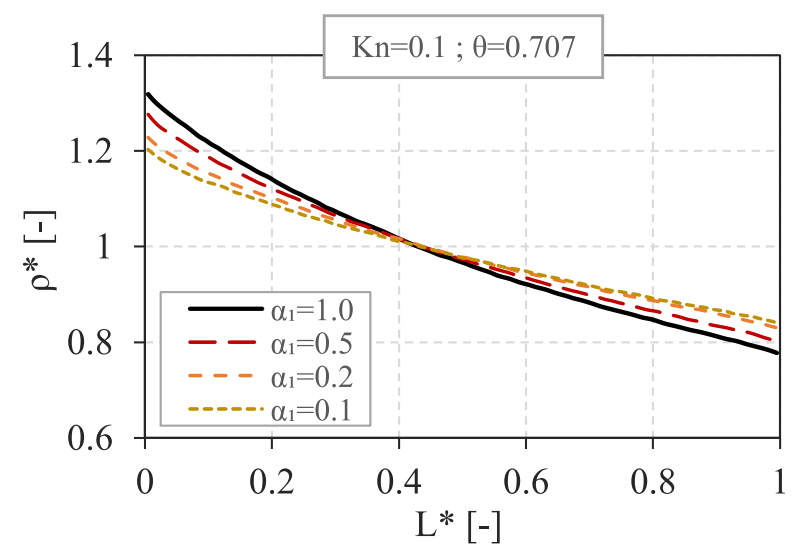

(b)

Figure 14: Dimensionless (a) temperature and (b) density profiles between the two surfaces for different values of accommodation coefficient at the adsorbing wall $\alpha_{1}$. Initial conditions: $K n=0.1, T_{w 2} / T_{w 1}=2, \tau_{0}^{*}=2 \cdot 10^{-4}, Q^{*}=10, \sigma=1$ and $\alpha_{2}=1$. For these initial conditions, the value of fractional coverage in steady-state regime is $\theta \approx 0.707$.

and the spatially one dimensional flow of a polyatomic gas is simulated between two plates held at different temperatures. At the first one, the developed absorption-desorption boundary condition is applied. The second wall, assumed to be impermeable, is described by Maxwell's gas-surface interaction model.

First, several simple tests are carried out to show the self-consistency of the proposed model. Then, the evolution of the surface coverage in time is analyzed in non-equilibrium conditions and the influence of several parameters, like the absorption time, the heat of absorption and the absorption probability, on flow properties is considered. In the case of the free molecular regime, the analytical solution is developed. The results show that the analytical and numerical solutions for free molecular regime presents deviation smaller than $0.3 \%$ for all evaluated cases.

\section{ACKNOWLEDGMENTS}

The authors would like to acknowledge financial supports provided by the European Union network program H2020, MIGRATE project under Grant Agreement No.643095. The second author S.S. would like to acknowledge also the partial financial support provided by Grant No BG05M2OP001-1.001-0003 under the Bulgarian SESG Operational Program (2014-2020) and co-financed by the European structural and investment funds.

\section{AppendixA. Analytical Solution for Free Molecular Flow Regime}

The analytical solution for free molecular regime was developed. The dimensionless density and temperature profiles can be calculated from Eqs. (A.1) and (A.2), respectively:

$$
\rho^{*}=\frac{\left(2-\alpha_{2}\right) \theta \rho_{w 1, \max }^{*}+\left[\left(2-\alpha_{2}\right) \beta \alpha_{1}+\left(1+\beta\left(1-\alpha_{1}\right)\right) \alpha_{2} \sqrt{\frac{T_{w 1}}{T_{w 2}}}\right] \rho_{w 1}^{*}}{2\left(1-\beta\left(1-\alpha_{1}\right)\left(1-\alpha_{2}\right)\right)}
$$




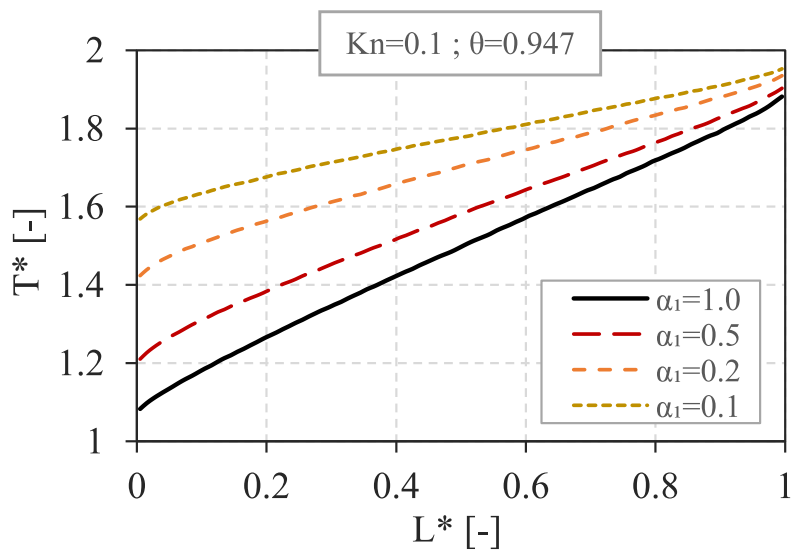

(a)

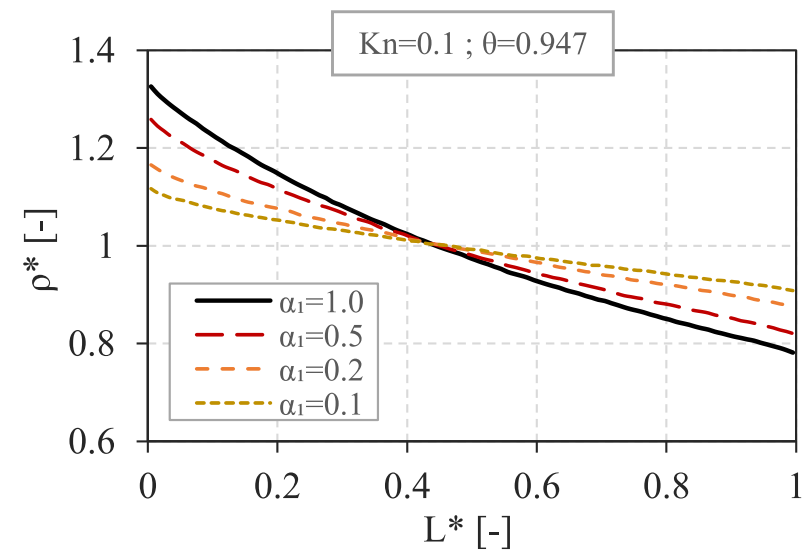

(b)

Figure 15: Dimensionless (a) temperature and (b) density profiles between the two surfaces for different values of accommodation coefficient at the adsorbing wall $\alpha_{1}$. Initial conditions: $K n=0.1, T_{w 2} / T_{w 1}=2, \tau_{0}^{*}=2 \cdot 10^{-4}, Q^{*}=12, \sigma=1$ and $\alpha_{2}=1$. For these initial conditions, the value of fractional coverage in steady-state regime is $\theta=0.947$.

$$
T^{*}=\frac{\left(2-\alpha_{2}\right) \theta \rho_{w 1, \max }^{*}+\left[\beta\left(2-\alpha_{2}\right) \alpha_{1}+\left(\beta\left(1-\alpha_{1}\right)+1\right) \alpha_{2} \sqrt{\frac{T_{w 2}}{T_{w 1}}}\right] \rho_{w 1}^{*}}{2 \rho^{*}\left(1-\beta\left(1-\alpha_{1}\right)\left(1-\alpha_{2}\right)\right)} .
$$

where

$$
\beta=1-(1-\theta) \sigma
$$

and

$$
\rho_{w 1, \max }^{*}=\frac{(1-\theta) \sigma}{\theta} \rho_{w 1}^{*} .
$$

The temperature and density profiles obtained by the numerical solution, represented by the solid black lines, and the analytical solution, indicated by the black $\mathbf{x}$ markers, are shown on Fig. 12. In the free molecular flow regime, the temperature and density are constant along the gap between the two surfaces.

The analytical solutions in the free molecular flow regime for the flow between two parallel fixed walls were obtained by other authors [7, 17], but without taking into account the absorption and desorption phenomena. From our analytical solution but considering no absorption $\left(\theta=1\right.$, therefore $\left.\rho_{w 1, \max }^{*}=0\right)$ and full accommodation for both surfaces $\left(\alpha_{1}=\alpha_{2}=1\right)$ we obtain the expressions provided in Refs. [7, 17].

[1] A. Frezzotti, G. P. Ghiroldi, L. Gibelli, Rerefied gas mixture flows driven by surface absoption, Vacuum 86 (2012) 1731-1738.

[2] A. Frezzotti, G. P. Ghiroldi, L. Gibelli, A. Bonucci, Dscm simulation of rarefied gas mixture flows driven by arrays of absorbing plates, Vacuum 103 (2014) 57-67.

[3] C. Tantos, S. Naris, D. Valougeorgis, Gas flow towards an adsorbing planar wall subject to partial gas-surface thermal accommodation, Vacuum 125 (2016) 65-74.

[4] H. J. Butt, K. Graf, M. Kappl, Physics and Chemistry of Interfaces, Wiley-VCH Verlag GmbH \& Co. KGaA, Weinheim, Germany, 2003, Ch. Adsorption, pp. 177-205. 


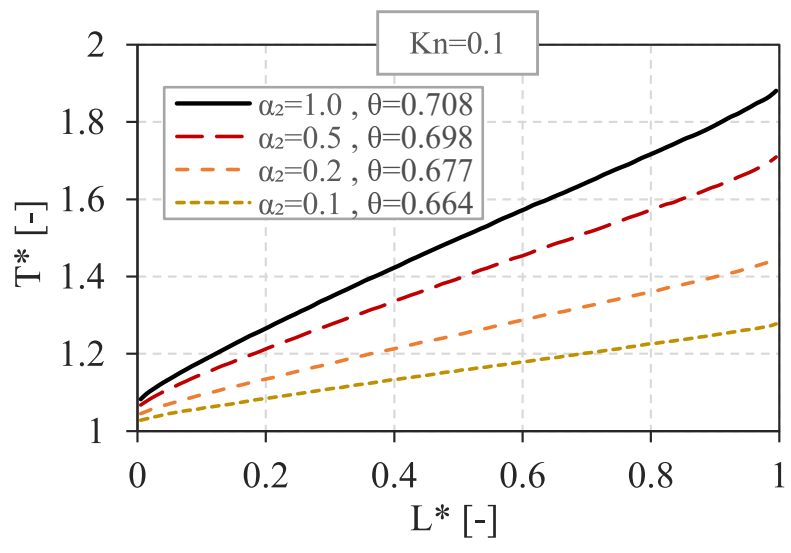

(a)

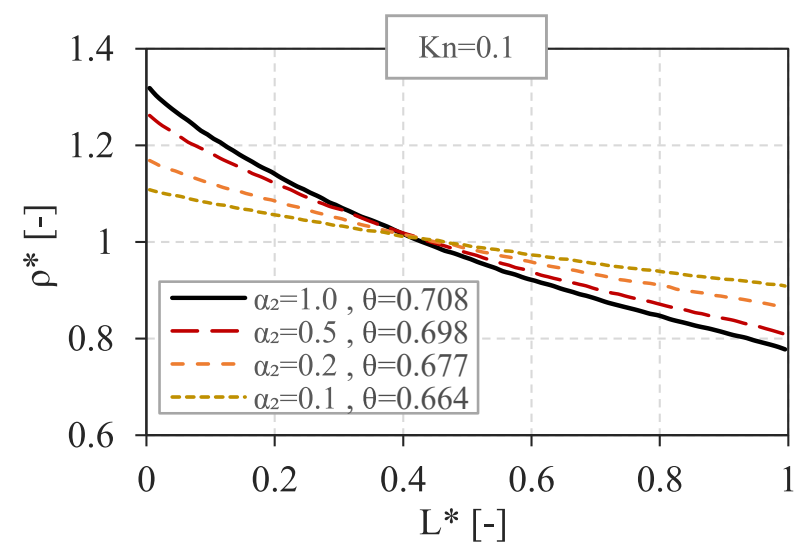

(b)

Figure 16: Dimensionless (a) temperature and (b) density profiles between the two surfaces for different values of accommodation coefficient at the reflecting wall $\alpha_{2}$. Initial conditions: $K n=0.1, T_{w 2} / T_{w 1}=2, \tau_{0}^{*}=2 \cdot 10^{-4}, Q^{*}=10, \sigma=1$ and $\alpha_{1}=1$.

[5] I. Langmuir, The construction and fundamental properties of solid and liquids, J. Am. Chem. Soc. 38 (11) (1916) 2221-2295.

[6] S. Brunauer, P. H. Emmett, E. Teller, Adsorption of gases in multilayers, J. Am. Chem. Soc. 60 (1938) 309-319.

[7] G. A. Bird, Molecular Gas Dynamics and the Direct Simulation of Gas Flows, Oxford Science Publications, Oxford University Press Inc., New York, 1994.

[8] C. Borgnakke, P. S. Larsen, Statistical collision model for monte carlo simulation of polyatomic gas hixtures, J. Comput. Phys. 18 (1975) 405.

[9] R. D. Brancher, S. Stefanov, A. Frezzotti, I. Graur, Kinetic theory description of gas adsorptiondesorption on a solid surface, in: AIP Conference Proceedings, Vol. 2132, 2019, pp. 170003, 1-9.

[10] T. Wolkenstein, Electron Theory of catalysis on Semiconductors, Pergamon. Oxford, 1963.

[11] C. Cercignani, Theory and application of the Boltzmann equation, Scottish Academic Press, Edinburgh, 1975.

[12] I. Kuščer, A model for rotational energy exchange in polyatomic gases, Physica A 158 (1989) 784-800.

[13] Y. Sone, Kinetic theoretical studies of the half-space problem of evaporation and condensation, Transport Theory Stat. Phys. 29 (3-5) (2000) 227-260.

[14] C. Tantos, G. P. Ghiroldi, D. Valougeorgis, A. Frezzotti, Effect of vibrational degrees of freedom on the heat transfer in polyatomic gases confined between parallel plates, International Journal of Heat and Mass Transfer 102 (2016) 162-173.

[15] E. Roohi, S. Stefanov, Collision partner selection schemes in DSMC: From micro / nano flows to hypersonic flows, Physics Reports 656 (March 2018) (2016) 1-38. 
[16] A. Frezzotti, T. Ytrehus, Kinetic theory study of steady condensation of a polyatomic gas, Physics of Fluids 18 (2006) 027101.

[17] B. D. Kay, D. W. Peden, C H F andGoodman, Kinetics of hydrogen absorption by Pd(110), Physical review B 34 (2) (1986) 817-822.

[18] I. A. Graur, A. Polikarpov, Comparison of different kinetic models for the heat transfer problem, Heat and Mass Transfer 46 (2009) 237-244. 


\begin{tabular}{ll}
\hline Subscript & name \\
\hline 0 & initial \\
$a d s$ & absorption \\
$d e s$ & desorption \\
$i n c$ & incident \\
$r$ & reflected \\
$r o t$ & rotational \\
transl & translational \\
$w 1$ & adsorbing wall \\
$w 2$ & reflecting wall \\
$x, y, z$ & x, y, z coordinates \\
$n$ & currently time step \\
$n+1$ & next time step \\
, & pre collision state \\
$*$ & dimensionless property \\
\hline
\end{tabular}

Table 3: List of subscripts and superscripts.

\begin{tabular}{llc}
\hline Sym & Variable & Unit \\
\hline$\alpha$ & accommodation coefficient & {$[-]$} \\
$\beta$ & beta coefficient & {$[-]$} \\
$\Gamma$ & gamma function & {$[-]$} \\
$\delta$ & delta function & {$[-]$} \\
$\Delta x$ & cell length & {$[\mathrm{m}]$} \\
$\Delta t$ & DSMC time step & {$[\mathrm{s}]$} \\
$\Delta t_{a}$ & absorption-desorption time step & {$[\mathrm{s}]$} \\
$\Delta \theta$ & variation of fractional surface coverage & {$[-]$} \\
$\theta$ & fractional surface coverage & {$[-]$} \\
$\lambda$ & mean free path & {$[\mathrm{m}]$} \\
$\rho$ & density & {$\left[\mathrm{kg} / \mathrm{m}^{3}\right]$} \\
$\sigma$ & absorption probability & {$[-]$} \\
$\tau$ & absorption or residence time & {$[\mathrm{s}]$} \\
$\tau_{0}$ & residence time constant & {$[\mathrm{s}]$} \\
$\tau_{a}$ & characteristic time of absorption & {$[\mathrm{s}]$} \\
$\tau_{e}$ & characteristic time of absorption and desorption & {$[\mathrm{s}]$} \\
$\phi$ & phi function & {$\left[1 /\left(\mathrm{m}^{3} . \mathrm{s}^{3}\right)\right]$} \\
\hline
\end{tabular}

Table 4: List of variables with greek alphabet. 


\begin{tabular}{|c|c|c|}
\hline Sym & Variable & Unit \\
\hline$A$ & area (of one binding site) & {$\left[m^{2}\right]$} \\
\hline$d$ & molecular diameter & {$[m]$} \\
\hline$f$ & Maxwellian wall function & {$\left[1 /\left(m^{6} . s^{3}\right)\right]$} \\
\hline$j$ & rotational degrees of freedom & {$[-]$} \\
\hline$J$ & molecular flux & {$\left[1 /\left(m^{2} . s\right)\right]$} \\
\hline$J_{i n c}$ & incident molecular flux & {$\left[1 /\left(m^{2} \cdot s\right)\right]$} \\
\hline$J_{m}$ & molecular flux impinging to the surface & {$\left[1 /\left(m^{2} \cdot s\right)\right]$} \\
\hline$J_{s c}$ & scattered back molecular flux & {$\left[1 /\left(m^{2} . s\right)\right]$} \\
\hline$k_{B}$ & Boltzmann constant & {$[J / K]$} \\
\hline$K_{a d s}$ & absorption constant & {$[1 /($ Pa.s $)]$} \\
\hline$K_{\text {des }}$ & desorption constant & {$[1 / s]$} \\
\hline$K_{L}$ & Langmuir constant & {$[1 / P a]$} \\
\hline$K_{L}^{\prime}$ & modified Langmuir constant & {$\left[m^{2} \cdot s\right]$} \\
\hline$K_{s c}$ & scattering kernel & {$[-]$} \\
\hline$K n$ & Knudsen number & {$[-]$} \\
\hline$L$ & length of domain & {$[m]$} \\
\hline$m$ & mass & {$[k g]$} \\
\hline$n$ & molecular number density or number of molecules per unit volume & {$\left[1 / m^{3}\right]$} \\
\hline$N$ & number of occupied sites per unit of area & {$\left[1 / m^{2}\right]$} \\
\hline$n_{a v}$ & number of DSMC time steps used in the average & {$[-]$} \\
\hline$N_{\Delta x}$ & number of cells & {$[-]$} \\
\hline$N_{\max }$ & total number of available sites per unit of area & {$\left[1 / m^{2}\right]$} \\
\hline$p$ & pressure & {$[P a]$} \\
\hline$Q$ & heat of absorption & {$[k J / m o l]$} \\
\hline$R_{a d s}$ & absorption rate & {$\left[1 /\left(m^{2} \cdot s\right)\right]$} \\
\hline$R_{\text {des }}$ & desorption rate & {$\left[1 /\left(m^{2} . s\right)\right]$} \\
\hline$t$ & time & {$[s]$} \\
\hline$t_{\max }$ & maximum time of simulation & {$[s]$} \\
\hline$t_{r}$ & reference time or gas relaxation time & {$[s]$} \\
\hline$T$ & temperature & {$[K]$} \\
\hline$u, v$ & velocity & {$[\mathrm{m} / \mathrm{s}]$} \\
\hline $\mathbf{u}, \mathbf{v}$ & macroscopic gas velocity & {$[\mathrm{m} / \mathrm{s}]$} \\
\hline$x, y, z$ & spatial coordinate & {$[m]$} \\
\hline$x_{\max }$ & length of the computational domain & {$[m]$} \\
\hline $\mathcal{E}$ & rotational energy & {$[J]$} \\
\hline $\mathcal{N}_{0}$ & initial number of simulated particles & {$[-]$} \\
\hline $\mathcal{N}_{0, c}$ & initial number of simulated particles per cell & {$[-]$} \\
\hline $\mathcal{R}$ & specific gas constant & {$[J /(k g . K)]$} \\
\hline $\mathcal{R}_{g}$ & gas constant & {$[J /($ mol.K $)]$} \\
\hline
\end{tabular}

Table 5: List of variables. 